\title{
Computing Stability Derivatives and Their Gradients for Aerodynamic Shape Optimization
}

\author{
Charles A. Mader* \\ University of Toronto, Toronto, Ontario M3H 5T6, Canada \\ and \\ Joaquim R. R. A. Martins \\ University of Michigan, Ann Arbor, Michigan 48109 \\ DOI: $\underline{10.2514 / 1 . J 052922}$
}

\begin{abstract}
Aerodynamic shape optimization of aircraft configurations often ignores stability considerations. To address this, a method for the computation of static, dynamic, and transient aircraft stability derivatives and their sensitivities for use in gradient-based optimization is introduced and evaluated. Computational fluid dynamics in the form of a three-dimensional structured-grid multiblock flow solver with both Euler and Reynolds-averaged Navier-Stokes equations is used. To compute the stability derivatives, a time-spectral formulation is used to compute an oscillating solution for the configuration of interest. From this oscillating solution, a series of linear regressions is performed to calculate the various stability derivatives. Because the solution is time dependent, it contains the information required to compute the transient, or "dot," derivatives for the configuration. An adjoint method is used to compute the gradients of the stability derivatives of interest, enabling gradient-based stability-constrained aerodynamic shape optimization with respect to a large number of design variables. The computed stability derivatives are verified for an airfoil and validated for a generic unmanned combat aerial vehicle. The stability-constrained optimization of a wing demonstrates the viability and usefulness of the method for aircraft design optimization.
\end{abstract}

\section{Nomenclature}

A

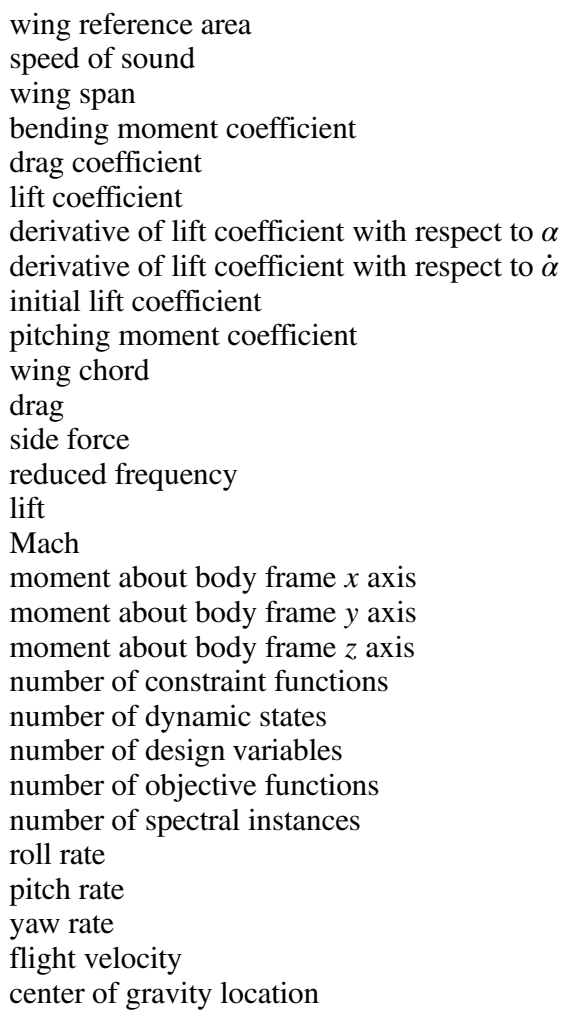

Received 4 July 2013; revision received 29 November 2013; accepted for publication 12 December 2013; published online 21 April 2014. Copyright $($ ) 2013 by the authors. Published by the American Institute of Aeronautics and Astronautics, Inc., with permission. Copies of this paper may be made for personal or internal use, on condition that the copier pay the $\$ 10.00$ per-copy fee to the Copyright Clearance Center, Inc., 222 Rosewood Drive, Danvers, MA 01923; include the code 1533-385X/14 and \$10.00 in correspondence with the CCC.

*Research Associate, Institute for Aerospace Studies. Member AIAA.

${ }^{\dagger}$ Associate Professor, Department of Aerospace Engineering. Associate Fellow AIAA.

$\begin{array}{lll}z_{e} & =\text { altitude } \\ \alpha & = & \text { angle of attack } \\ \dot{\alpha} & = & \text { time derivative of } \alpha \\ \beta & = & \text { side-slip angle } \\ \Delta \alpha & = & \text { change in } \alpha \\ \Delta \mathbf{y}_{\mathrm{FFD}} & = & \text { free form deformation volume control } \\ \theta_{i} & =\text { point movement in } y \text { direction } \\ \lambda & =\text { wing sweep } \\ \phi & =\text { mesh rotation angle about the } z \text { axis }\end{array}$

\section{Introduction}

$\mathbf{C}$ OMPUTATIONAL fluid dynamics (CFD) based aerodynamic shape optimization has evolved considerably in the last few decades. Using adjoint methods with Euler and Navier-Stokes CFD, a number of researchers have successfully solved drag minimization problems [1-6] for a variety of aircraft configurations and for problems in which the aerodynamic shape was optimized simultaneously with the structural sizing, leading to optimal aeroelastic tailoring $[\underline{7}, \underline{8}]$. However, when considering full aircraft configurations there is a coupling between the aerodynamic efficiency of the aircraft and its trim and stability characteristics. In the case of conventional aircraft, the moment characteristics of the wing, as well as the configuration of the wing and tail with respect to the aircraft center of gravity (CG) impact the amount of trim drag generated by the tail. In the case of a tailless aircraft, the planform and airfoil shape of the wing are integrally related to the trim, stability, and performance of the design.

Therefore, to achieve feasible solutions that offer an optimal tradeoff between the aerodynamic performance and stability considerations, we need to compute the stability derivatives and implement them as constraints in aerodynamic shape optimization problems. To address this need, we develop a method for computing static and dynamic stability derivatives, and their gradients, that enables gradient-based stability-constrained aerodynamic shape optimization. Although the computation of stability derivatives has been studied extensively, as detailed in the next section, the implementation of stability derivative constraints in aerodynamic shape optimization problems has not. Given that the stability constraints are themselves derivative quantities, it is challenging to compute the gradients of these quantities efficiently. 
The objective of the present work is to develop an approach for which the computation of the gradients of the stability derivatives is efficient and accurate enough to enable stability-constrained aerodynamic shape optimization with respect to large numbers of design variables. The method presented herein has already been used to conduct a study on the effect of stability considerations in the optimal shape of flying wings [5]. However, that previous study focused on comparing the results of the various stability-constrained optimization problems, not on the method for computing the stability derivative constraints. This work presents the details of that method, including a validation of the stability derivative computations and discussion on the computational tradeoffs of various methods for computing stability derivatives when used in gradient-based optimization.

We start this paper by reviewing the current state of the art in stability derivative computation in Sec. II, followed by a discussion of the additional considerations that are required for optimization in Sec. III. We then introduce our approach to the computation of stability derivatives in Sec. IV, highlighting the major differences with respect to existing methods and presenting two validation cases to demonstrate the effectiveness of the method. Finally, in Secs. Vand

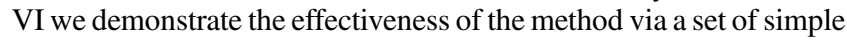
optimizations that include stability derivatives as constraints.

\section{Background}

There are two main approaches to computing stability derivatives with CFD. The first involves applying a conventional derivative computation, such as finite differencing, automatic differentiation (AD), or adjoint methods, to a steady flow solution. Applying this approach to a conventional CFD formulation allows the computation of the static stability derivatives. These techniques have been demonstrated by several authors. Charlton [9] conducted simple $\alpha$ and $\beta$ sweeps to get the force and moment information required for falling-leaf predictions for tailless aircraft. Godfrey and Cliff [10] explored the use of analytic sensitivity methods, in particular the direct method, for the computation of static stability derivatives. Park et al. [11] applied ADIFOR [12], an AD tool, to a three-dimensional viscous flow solver to compute the static derivatives of various configurations.

To compute the dynamic derivatives using these techniques, the CFD formulation needs to be modified to include the dynamic parameters. These approaches have also been well explored. Park and Green [13] extended their study on using AD to compute stability derivatives to include the dynamic derivatives. Limache and Cliff [14] demonstrated the use of adjoint methods for the computation of dynamic stability derivatives for a two-dimensional case. Babcock and Arena [15] modified the boundary conditions in a finite element based Euler CFD solver to separate the velocity and position boundary conditions and to allow the computation of the dynamic derivatives using finite differencing. Mader and Martins [16] demonstrated the use of an $\mathrm{AD}$ adjoint solver for the computation of stability derivatives on a three-dimensional CFD solver. This class of approaches, based on steady CFD solutions, is efficient for computing the various stability derivatives because only a single steady solution is required in addition to the chosen derivative computation.

The second general approach involves computing the solution of a forced oscillation of the aircraft, then using that unsteady solution to estimate the stability derivatives. In this approach, multiple flow solutions are required to compute a full set of derivatives. The exact number of solutions depends on the specific motions used for the forced oscillation solution as well as the techniques used to analyze that solution. This approach has been examined by a variety of researchers. Early work on this topic was conducted mostly with respect to missiles and projectiles. Several researchers, including Weinacht [17], DeSpirito et al. [18], Sahu [19], and Oktay and Akay [20], demonstrated methods using steady and unsteady CFD to predict the stability derivatives of missiles and projectiles. More recently, Murman [21] presented a method for computing stability derivatives of both missiles and full aircraft configurations using a frequency-domain CFD method. He used the frequency domain solver to produce periodic data for the forced oscillation of the configuration of interest. The data were then analyzed with the same techniques used to produce stability derivatives from forcedoscillation wind-tunnel data, which allowed the method to take advantage of the large body of knowledge in that field. A number of papers from the recent NATO Research and Technology Organisation Task Group AVT-161 have explored the use of forced oscillation techniques with a variety of CFD solvers [22], including Reynolds-averaged Navier-Stokes (RANS) [23,24], direct eddy simulation [25,26], and harmonic balance [27] solvers. The results were shown to correlate well with experimental data. Additional details regarding some of these results have been presented by $\mathrm{Da}$ Ronch et al. [28, 29].

\section{Considerations for Optimization}

Using the stability derivatives in an optimization adds another layer of complexity to the selection of an appropriate computational method. The method not only needs to be efficient for computing the stability derivatives, but also needs to provide efficient computation of the gradients of the stability derivatives with respect to the design variables in order to allow for gradient-based optimization. The methods based on steady flow requirements certainly satisfy the first criterion. However, methods that rely on advanced derivative techniques (such as the adjoint method) for computing the stability derivatives significantly complicate the computation of the optimization gradients. In these cases, the gradients of the stability derivatives become second-order derivatives and are very costly to compute. It is possible to compute them relatively efficiently using a second-order adjoint method such as that proposed by Ghate and Giles [30] or Rumpfkeil and Mavriplis [31]. However, the cost of this method scales with the sum of the number of objectives, constraints, and design variables (outputs plus inputs). It quickly becomes prohibitive when large numbers of design variables are used. This limitation can be relieved somewhat by using finite differences to compute the stability derivatives. In this case, an extra flow solution (and set of adjoint solutions) is required for each individual stability parameter $(\alpha, \beta, V, p, q, r$, etc.). Although this method is again independent of the number of design variables, it increases the total number of flow and adjoint solutions required for a given iteration. This method has been used for simple static margin constraints in aerostructural optimization by Kenway and Martins [7] and Liem et al. [32].

The other significant drawback of all of the steady methods is that they contain no time-dependent information in the solution. Therefore, with the exception of some special cases in which $\dot{\alpha}$ can be excited using a helical motion [17], it is not possible to compute the transient derivatives necessary for the linear flight dynamics model. Unfortunately, computing the stability derivatives with a full timedependent solution in order to include that information would be extremely expensive. Several authors have examined the use of adjoint methods in time-dependent optimizations, both in two dimensions [33-35] and three dimensions [36,37]. Although the time-dependent adjoint method is certainly an improvement over finite difference sensitivity methods, it still incurs a high computational cost. Fortunately, because of the periodic nature of the solutions we use to compute the stability derivatives, we are able to use spectral CFD methods to reduce the cost of both the computation and the associated adjoint method for computing optimization gradients. The efficiency of adjoint-based shape optimization using spectral methods has been demonstrated previously by Nadarajah and Jameson [38], who optimized an oscillating transonic wing using an adjoint implementation for the nonlinear frequency domain (NLFD) equations, and by Choi et al. [39], who used an adjoint method for the time-spectral method to optimize a helicopter rotor blade. These previous results indicate that an approach based on timespectral CFD methods will be efficient in this case also.

Using time-spectral CFD, the cost of computing the stability derivatives scales with the number of spectral instances required to capture the unsteady effects in the oscillating solution as well as the number of motions that need to be simulated to excite the various 
dynamic states of interest. The cost of the adjoint solutions for the derivatives scales in the same fashion. Therefore, the scaling of this method is similar to the scaling of the steady method where finite differencing is used to compute the stability derivatives. Table 1 summarizes the relative costs of the two steady approaches and the pseudo-steady spectral approach for computing the stability derivatives and their gradients.

For the optimizations presented here, we have one objective $\left(C_{D}\right)$, three or four CFD constraints, and approximately 290 design variables. We are concerned only with the $\alpha$ derivatives, which means we can simulate all of the required derivatives by exciting a single dynamic state. Therefore, the cost of method 1 would be $1+1+$ $3+290=295$ equivalent flow solutions, the cost of method 2 would be $(1+1+3) \times(1+1)=10$, and the cost of method 3 would be $(3 \times 1)(1+1+3)=15$. Thus, the unsteady method is only marginally more expensive than the second steady method, and it allows us to compute the $\dot{\alpha}$ derivatives. Therefore, it is the method chosen here. Note that because of the simple motions involved in this case we are able to limit the number of spectral instances to three, which reduces the cost of the unsteady method. We have also assumed that the cost of an adjoint solution is approximately the same as the cost of a flow solution.

\section{Theory}

The time-spectral stability derivative formulation presented in the following section is similar to the methods presented by Murman [21] and $\mathrm{Da}$ Ronch et al. [27,29] in that it uses a time-periodic approximation to the unsteady $\mathrm{CFD}$ solution to reduce the cost of the computation. However, the forced oscillation motions used here and the linear regression approach used to determine the derivatives in this work differ somewhat from those previously used. These two components are motivated by the complex number $\dot{\alpha}$ derivative methodology outlined by Etkin [40] and are based on the idea that the transient derivatives are related to the time lag in the development of the aerodynamic forces when an aircraft changes orientation. A similar linear regression method for determining the stability derivatives is used with experimental flow results by Rohlf et al. [41].

As with other time-periodic CFD stability derivative techniques, the time-spectral stability derivative method is essentially a forced oscillation technique. A time-spectral CFD solver is used to generate the solution for a prescribed oscillatory motion. This periodic solution is then used with a linear regression technique to generate estimates for the functionals of interest: the force or moment coefficient, the derivative of that coefficient with respect to the oscillating parameter, and the derivative of that coefficient with respect to the time derivative of the oscillating parameter. In this work, we focus on using relatively small-amplitude oscillations, less than $2 \mathrm{deg}$, at low reduced frequencies, with single-parameter oscillations to isolate specific derivatives. The simple algebraic nature of the method allows it to be used in conjunction with an adjoint method to compute the gradients necessary for aerodynamic shape optimization, thereby allowing for efficient optimization with respect to large numbers of design variables.

\section{A. Time-Spectral CFD}

The underlying core of this approach to computing stability derivatives is the time-spectral CFD method. Early work on timenonlinear spectral solution techniques was conducted by Hall et al. [42], who derived a spectral formulation for the two-dimensional Navier-Stokes equations. In an extension of this work, Ekici and Hall [43] applied the technique, known as the harmonic balance technique, to multistage turbomachinery applications in which a variety of frequencies may be present. Other spectral methods have been demonstrated by McMullen et al. [44,45] (the NLFD method) and Gopinath and Jameson [46] (the time-spectral method). The main differences between the methods are the portions of the solution that are computed in the frequency and time domains.

In this work, we use the time-spectral implementation in the SUmb flow solver [46-49]. This implementation is based on the timedomain version of the equations as derived by Gopinath et al. [46,50] and Van der Weide et al. [48]. The flow equations are discretized with a second-order finite volume scheme with scalar dissipation. The rigid body grid motion is implemented with an Arbitrary LagrangianEulerian scheme. For a complete review of the method, we refer the reader to the references.

\section{B. Linearized Aerodynamic Forces}

We base our stability derivative method on the linear air reaction theory outlined by Etkin [40]. From this theory, we know that for a general motion the force and moment coefficients of an aircraft, for example, the lift coefficient, can be approximated as

$$
\begin{aligned}
C_{L}= & C_{L_{0}}+C_{L_{\alpha}} \Delta \alpha+C_{L_{\dot{\alpha}}} \Delta \dot{\alpha}+C_{L_{\ddot{\alpha}}} \Delta \ddot{\alpha}+\ldots+C_{L_{\beta}} \Delta \beta \\
& +C_{L_{\dot{\beta}}} \Delta \dot{\beta}+\ldots
\end{aligned}
$$

where all the motion states $\alpha, \beta, V, p, q, r, z_{e}$ and their associated time derivatives are included, and $C_{L_{0}}$ is the value of the coefficient for the steady-state reference flight condition about which the motion occurs. However, if a simple motion is specified that consists of a single dynamic state, for example, $\alpha$, the values of the remaining motion states are zero and the associated derivatives drop out of the equation. In the case of a pure $\alpha$ motion this leads to

$$
C_{L}=C_{L_{0}}+C_{L_{\alpha}} \Delta \alpha+C_{L_{\dot{\alpha}}} \Delta \dot{\alpha}+C_{L_{\ddot{\alpha}}} \Delta \ddot{\alpha}+\ldots
$$

One can then make the further assumption, justified in the following discussion, that the higher-order derivatives are small and can be neglected, which gives

$$
C_{L} \approx C_{L_{0}}+C_{L_{\alpha}} \Delta \alpha+C_{L_{\dot{\alpha}}} \Delta \dot{\alpha}
$$

or in a more general form

$$
C_{i} \approx C_{i_{0}}+C_{i_{j}} \Delta j+C_{i_{j}} \Delta \dot{j}
$$

where $i=L, D, F_{y}, M_{x}, M_{z}, M_{y}$ and $j=\alpha, \beta, V, p, q, r$. Based on the simplified linearization in Eq. (4), we are now left with a simple equation with three unknowns, $C_{i_{0}}, C_{i_{j}}$, and $C_{i_{j}}$.

\section{Prescribed Motion}

As we can see from the previous section, the key requirement for identifying the values of individual stability derivatives is the simulation of a motion that excites a single dynamic state and its higher-order derivatives. Several researchers have examined ways to separate pitching and plunging motions, particularly in the context of analyzing projectiles and missiles. Both Qin et al. [51] and Weinacht [17] introduce methods for calculating the $q$ and $\dot{\alpha}$ derivatives based on steady coning and helical motions. However, these motions include nonzero roll and yaw rates, respectively, which could cause problems in the context of nonaxisymmetric bodies such as full aircraft configurations. Weinacht also introduces the idea of using a steady looping motion to get the $q$ derivatives. This motion is suitable for use with aircraft and has been demonstrated in that context by

Table 1 Stability derivative methods: equivalent computational cost comparison

\begin{tabular}{lccccc}
\hline \hline Method no. & Stab. deriv. method & Gradient method & Equiv. flow solutions & Equiv. adjoint solutions & Total cost \\
\hline 1 & Adjoint & Second-order adjoint & 1 & $n_{\mathrm{obj}}+n_{\mathrm{con}}+n_{\mathrm{dv}}$ & $1+n_{\mathrm{obj}}+n_{\mathrm{con}}+n_{\mathrm{dv}}$ \\
2 & FD & Adjoint & $1+n_{\mathrm{ds}}$ & $\left(n_{\mathrm{obj}}+n_{\mathrm{con}}\right)\left(1+n_{\mathrm{ds}}\right)$ & $\left(1+n_{\mathrm{obj}}+n_{\mathrm{con}}\right)\left(1+n_{\mathrm{ds}}\right)$ \\
3 & TS & TS Adjoint & $n_{\mathrm{sp}} n_{\mathrm{ds}}$ & $n_{\mathrm{sp}} n_{\mathrm{ds}}\left(n_{\mathrm{obj}}+n_{\mathrm{con}}\right)$ & $\left(n_{\mathrm{sp}} n_{\mathrm{ds}}\right)\left(1+n_{\mathrm{obj}}+n_{\mathrm{con}}\right)$ \\
\hline \hline
\end{tabular}


several authors $[13,14,16]$. However, as discussed in Sec. II, methods based on this approach do not lend themselves to optimization. Because we have chosen to use an unsteady forced-oscillation approach to compute the stability derivatives, we have chosen to use pitching and plunging motions to compute the separated stability derivatives. The motions that we aim to recreate are those illustrated on page 267 of Etkin [40]. Note that these are also equivalent to the two-dimensional projections of the helical motions outlined by Weinacht [17]. However, these specific motions are difficult to realize physically and require some modification to a standard CFD solver to implement computationally.

For the $\alpha$ motion, we specify a standard oscillating value of $\alpha$ :

$$
\alpha=A \sin (\omega t)
$$

However, in addition to this, we alter the velocity of the grid to be

$$
\begin{gathered}
V_{x}=a M \cos (\alpha) \\
V_{y}=-a M \sin (\alpha) \\
V_{z}=0
\end{gathered}
$$

so that the magnitude of the velocity stays constant and any Mach effects are removed from the oscillation. For the $q$ motion, we again use a standard pitch rate oscillation:

$$
\begin{gathered}
\phi=A \sin (\omega t) \\
q=\mathrm{d} \phi=A \omega \cos (\omega t)
\end{gathered}
$$

However, in this case, we modify the grid velocity to maintain a constant angle of attack throughout the oscillation, giving

$$
\begin{gathered}
V_{x}=a M(\cos (\alpha) \cos (\phi)-\sin (\alpha) \sin (\phi)) \\
V_{y}=-a M(\cos (\alpha) \sin (\phi)+\sin (\alpha) \cos (\phi)) \\
V_{z}=0
\end{gathered}
$$

When one of these pure motions is simulated with the time-spectral method, the result is a solution that consists of force coefficient $\left(C_{L}\right.$, $\left.C_{D}, C_{F_{y}}\right)$ and moment coefficient $\left(C_{m_{x}}, C_{m_{y}}, C_{m_{z}}\right)$ values at $N$ time instances in a periodic solution. A set of solutions corresponding to a pure $\alpha$ motion is shown in Fig. 1 .

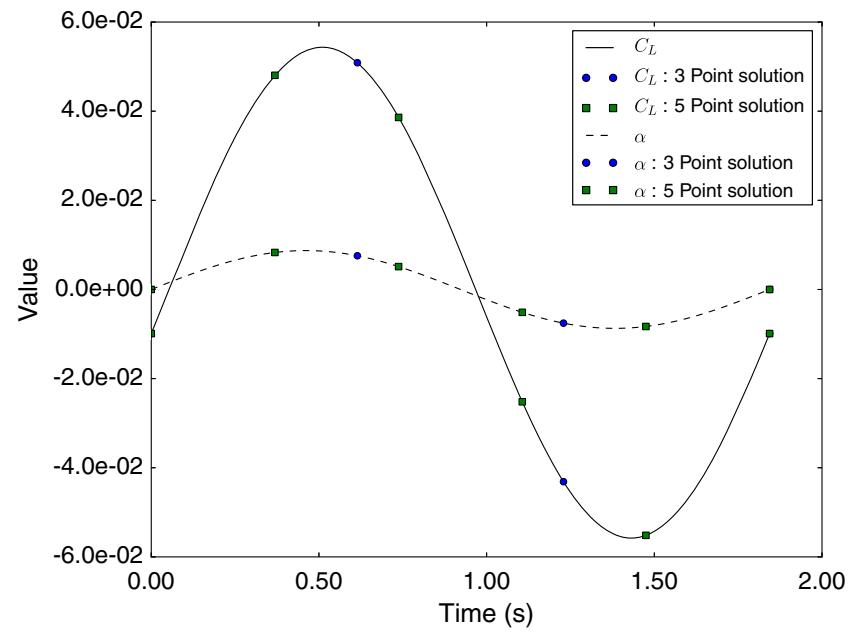

Fig. 1 Time-spectral solution: $\Delta \alpha$ motion at $M=0.1$.
Now that we have a periodic time history of the coefficient with respect to the oscillating parameter, we can use this information to compute the various stability derivatives with respect to that parameter. To accomplish this, we relate the coefficients to the oscillating parameter through the time variable. This leads to the relationship shown in Fig. 2 . As the figure shows, the general trend of the relationship between the coefficient and the motion parameter is linear. However, there is also a distinct hysteresis in the solution, as demonstrated by the gap between the coefficient on the upstroke and downstroke of the oscillation. This hysteresis relates to the transient or "dot" derivatives. As discussed by Etkin [40] with respect to the $\dot{\alpha}$ derivatives, the transient derivatives represent the time lag in the development of the coefficient resulting from a change in the flow. This idea leads us to the method used to compute the stability derivatives.

To compute stability derivatives from this solution, one computes a linear least-squares fit of the output coefficient (e.g., $C_{L}$ ) with respect to the primary motion variable from the solution (e.g., $\alpha$ ). The slope of the line resulting from this fit, shown in Fig. $\underset{2}{2}$, is the stability derivative $\left(C_{L_{\alpha}}\right)$, whereas the $y$ intercept of the line is the value of the coefficient $\left(C_{L}\right)$ at the zero value of the motion perturbation $\Delta \alpha=0$. This yields an approximation of the form

$$
y=C_{i_{j}} x+C_{i_{0}}
$$

We now take advantage of the hysteresis in the solution to calculate the transient derivatives. To estimate this quantity, we subtract the value of the linear regression line from the time-spectral solution:

$$
R_{C_{i}}^{n}=C_{i}^{n}-y\left(x^{n}\right)
$$

This process eliminates the bulk dependence of the solution on the main motion variable, leaving just the variation $\left(R_{C_{i}}^{n}\right)$ associated with the hysteresis. This yields another strongly linear relationship, as shown in Fig. 3. Taking the slope of this trend yields the value of the transient derivative $\left(C_{i_{\dot{\alpha}}}\right)$. Further, as this plot shows, the linear approximation is a very good representation of the $\dot{\alpha}$ trend, indicating that any dependence on the higher-order time derivatives is small. This is sufficient to justify the assumption made for Eq. (3). Also note that Figs. $\underline{1}$ and $\underline{2}$ demonstrate that three time instances are sufficient to model the forced-oscillation solution. In these figures, the extra points in the five- and seven-instance cases lie on the same sinusoidal solution predicted by the three-instance solution. This is consistent with the findings of Murman [21]. Murman observes that the ability to simulate the forced oscillation with a single frequency arises because the forcing frequency of the oscillation largely determines the frequency of the output and because there is a limited potential for energy transfer between the modes when using the Euler equations. We also note that, as demonstrated by Rohlf et al. [41], smaller amplitude oscillations tend to produce more sinusoidal, if also noisier,

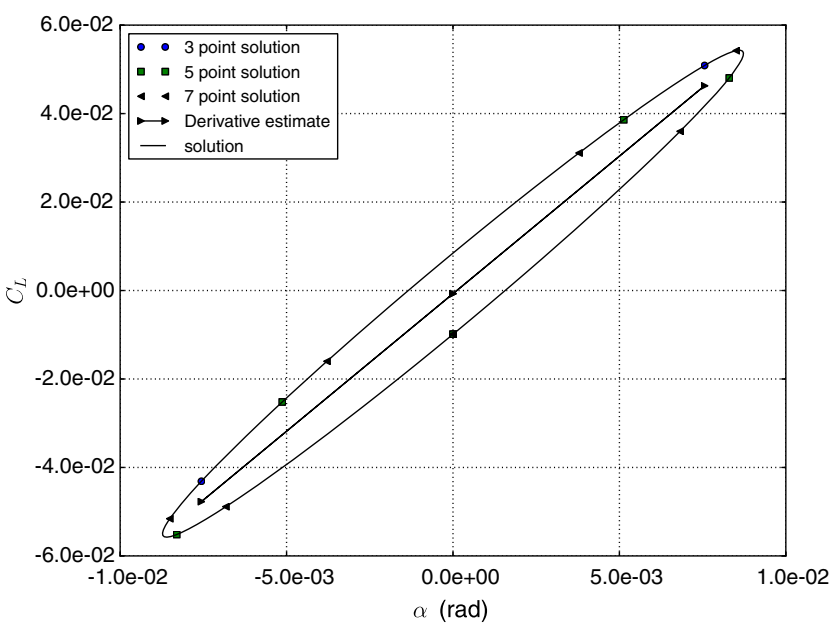

Fig. 2 Time-spectral solution: $C_{L}$ vs $\alpha$ at $M=0.1$. 


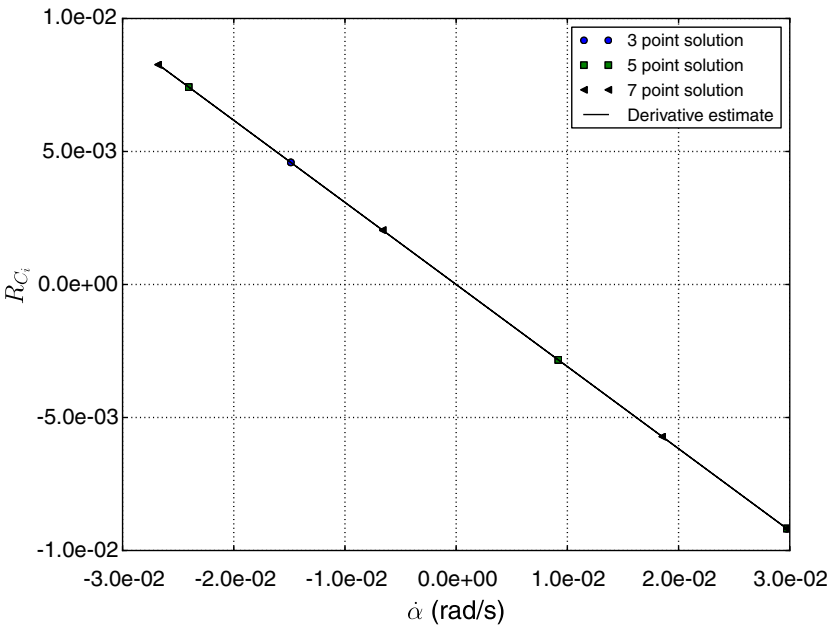

Fig. 3 Time-spectral solution: $C_{L}$ hysteresis vs $\dot{\alpha}$ at $M=0.5$.

output in the experiments. Because noise is not a major issue for timeperiodic CFD solutions, we have chosen to use small-amplitude oscillations in this work, on the order of 0.5 to $2 \mathrm{deg}$.

With this simple algebraic stability derivative computation in place, it is now a simple extension to use the time-spectral adjoint method of Mader and Martins [52] to compute the gradients of this approach for shape optimization. The time-spectral adjoint approach outlined by Mader and Martins uses simple agglomeration functions to combine the $N$ spectral force coefficients into a single value for the adjoint function. In the simplest case this can be just an averaging of the coefficients:

$$
C_{i}=\frac{1}{N} C_{i_{1}}+\frac{1}{N} C_{i_{2}}+\ldots+\frac{1}{N} C_{i_{N}}
$$

In our case, the linear regression operation described previously forms the basis of this agglomeration function. Therefore, using this approach with the time-spectral adjoint simply requires a minor modification to the partial derivatives of the objective function. Full details on the implementation of the time-spectral adjoint can be found in the authors' previous work [52].

\section{Verification and Validation}

To verify and validate the time-spectral stability derivative approach, we examine two test cases. The first is a NACA 0012 airfoil undergoing an oscillating plunging motion, and it is compared with a theoretical thin-airfoil theory result. The second is the stability and control configuration (SACCON) uninhabited combat air vehicle (UCAV), which is compared with wind-tunnel results.

\section{NACA 0012: Test Case Description}

The NACA 0012 case is a two-dimensional case that we emulate using our three-dimensional solver. The meshes have only two cells in the spanwise direction, with symmetry planes on both sides to simulate two-dimensional flow. The mesh is a C-mesh topology split into 16 blocks for efficient parallel balancing, as shown in Fig. 4a. The near field around the airfoil is shown in Fig. 4b. This set of test cases is simulated using the Euler equations at $M=0.1$.

To assess the numerical accuracy of the solutions, we conducted a mesh convergence study with meshes containing 4096, 16,384, and 65,536 cells per slice. The mesh convergence results are shown in Table 2.

The values of the coefficients $\left(C_{L}, C_{m}\right)$ and their derivatives with respect to $\alpha$ show excellent numerical accuracy, with relative errors less than $1 \%$ on the finest mesh for both sets of coefficients and derivatives. The accuracy of the derivatives with respect to $\dot{\alpha}$ is slightly lower, with relative errors on the order of $2 \%$ on the finest mesh. However, this level of error is acceptable for our purposes.

\section{NACA 0012: Verification}

To verify our implementation of the time-spectral stability derivative method, we compare the NACA 0012 results to those from thin-airfoil theory. Etkin [40] presents theoretical results for an oscillating plunging airfoil, based on the work of Theodorsen. Using the Theodorsen function as a basis, the theoretical values of the lift and moment coefficients can be expressed as

$$
C_{L_{\alpha}}=2 \pi F(k)
$$

$$
C_{L_{\dot{\alpha}}}=\pi+2 \pi \frac{G(k)}{k}
$$

$$
C_{m_{\alpha}}=2 \pi F(k)\left(h-\frac{1}{4}\right)
$$

$$
C_{m_{\dot{\alpha}}}=\pi\left(h-\frac{1}{2}\right)+2 \pi \frac{G(k)}{k}\left(h-\frac{1}{4}\right)
$$
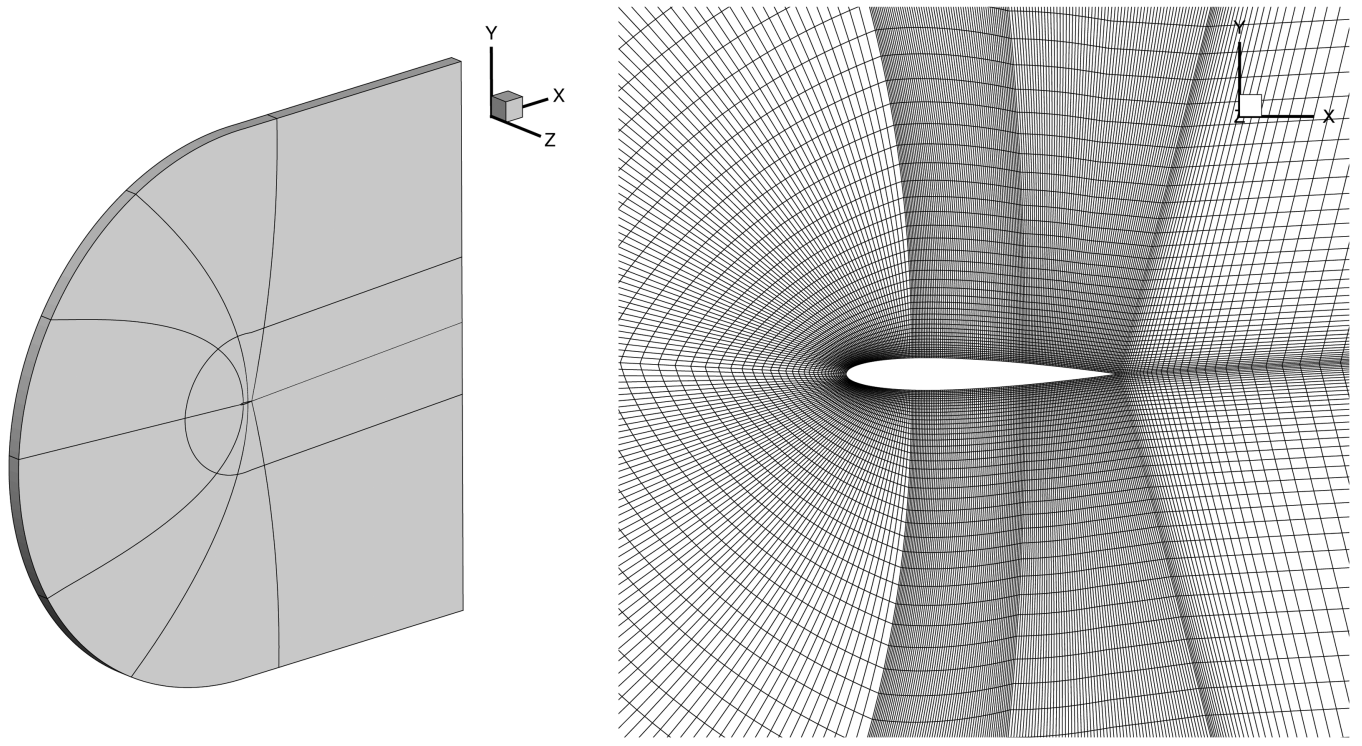

a) Mesh topology

b) Near field mesh: 65536 cells per slice

Fig. 4 NACA 0012 mesh. 
Table 2 NACA 0012 mesh refinement results

\begin{tabular}{lcccccc}
\hline \hline Case size (cell/slice) & $C_{L}$ & $C_{m}$ & $C_{L_{\alpha}}$ & $C_{m_{\alpha}}$ & $C_{L_{\dot{\alpha}}}$ & $C_{m_{\dot{\alpha}}}$ \\
\hline 4096 & 0.1896 & -0.0461 & 5.0660 & -1.2745 & -12.4044 & 2.2542 \\
16,384 & 0.2377 & -0.0621 & 6.1827 & -1.6199 & -20.6218 & 4.8321 \\
65,536 & 0.2397 & -0.0625 & 6.2298 & -1.6217 & -21.4835 & 5.1051 \\
Estimate (Richardson extrapolation) & 0.2404 & -0.0626 & 6.2455 & -1.6223 & -21.7708 & 5.1961 \\
65,536 cell \% error & 0.283 & 0.163 & 0.252 & 0.0367 & 1.319 & 1.751 \\
16,384 cell \% error & 1.134 & 0.653 & 1.006 & 0.1467 & 5.277 & 7.004 \\
\hline \hline
\end{tabular}

Here $F(k)$ and $G(k)$ are the real and imaginary parts of the Theodorsen function [53],

$$
C(k)=F(k)+i G(k)
$$

and $h$ is the percent chord at which the moment coefficient $\left(C_{m}\right)$ is evaluated. The four plots in Fig. 5 show a comparison of the lift and moment coefficient derivatives with respect to $\alpha$ and $\dot{\alpha}$ as predicted by the theoretical model and the time-spectral model. Because the theoretical result varies with the frequency, the figure also shows the variation in the derivative over a range of reduced frequencies. The data points on the plot represent the discrete reduced frequencies at which the time-spectral solutions were analyzed. In addition, because the theoretical result is for an infinitely thin airfoil, we computed numerical results for the full-thickness airfoil as well as half- and quarter-thickness airfoils. As shown in Fig. $\underline{5}$, the numerical results match the theoretical results well over the full range of reduced frequencies. There is a small offset between the numerical results and the theoretical results. However, for the $\dot{\alpha}$ derivatives, this discrepancy reduces significantly for the half- and quarter-thickness airfoils, indicating that most of it comes from the thin-airfoil assumptions of the reference results. For the $\alpha$ derivatives, the trend is less conclusive. At lower frequencies, the thin results match the theoretical result more closely than the full-thickness results, whereas at higher frequencies the offset is larger for the thin airfoils than for the thick airfoils. However, the shape of the curve for the thinner airfoil results matches the theoretical result more closely than does the shape of the thicker airfoils. Note that the theoretical results for the $\dot{\alpha}$ derivatives become undefined as the reduced frequency tends to zero. This singularity is clearly apparent in Eqs. (18) and (20), and it prevents the calculation of a definitive value for the derivative. However, given the demonstrated agreement between the theoretical and numerical results, this comparison confirms that the timespectral stability derivative method produces correct results for this simple two-dimensional case.

\section{SACCON UCAV: Test Case Description}

The second test case considered is the SACCON, a flying-wing, UCAV configuration. The mesh is constructed with an O topology and 13 blocks. The geometry of the case is based on the information provided in Schütte et al. [54] and Tormalm and Schmidt [26]. We analyze the flow around the UCAV using a RANS flow solver [47]
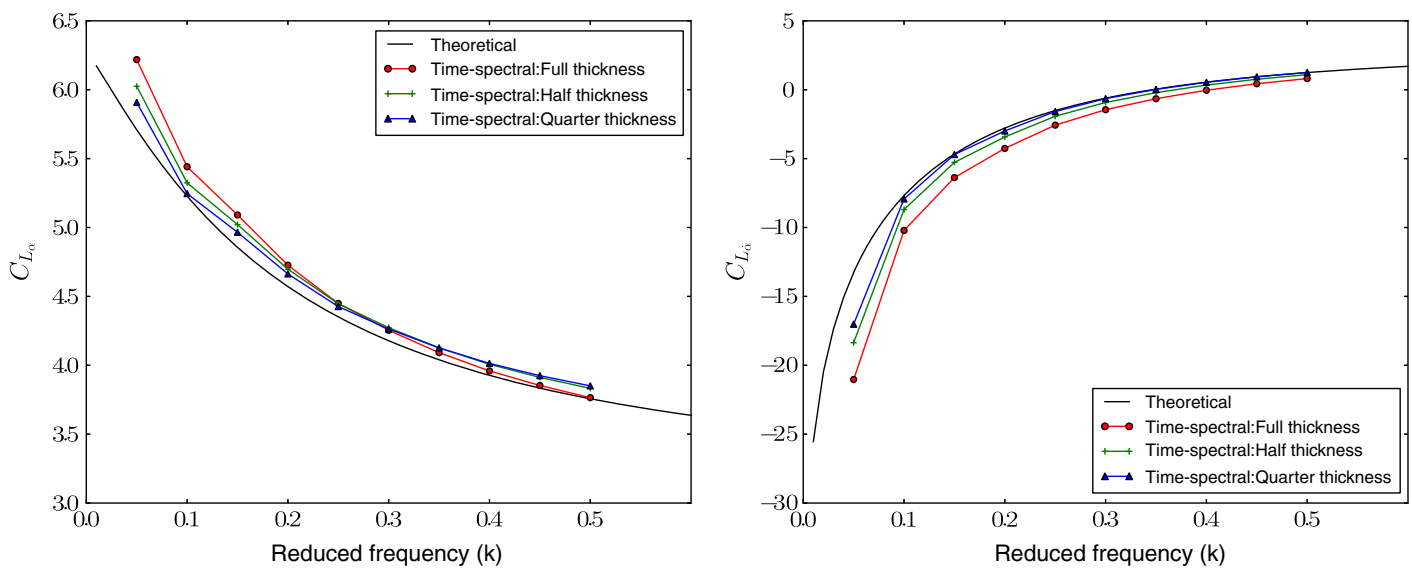

a) $\boldsymbol{C}_{\boldsymbol{L}_{\alpha}}$

b) $C_{L_{\dot{\alpha}}}$
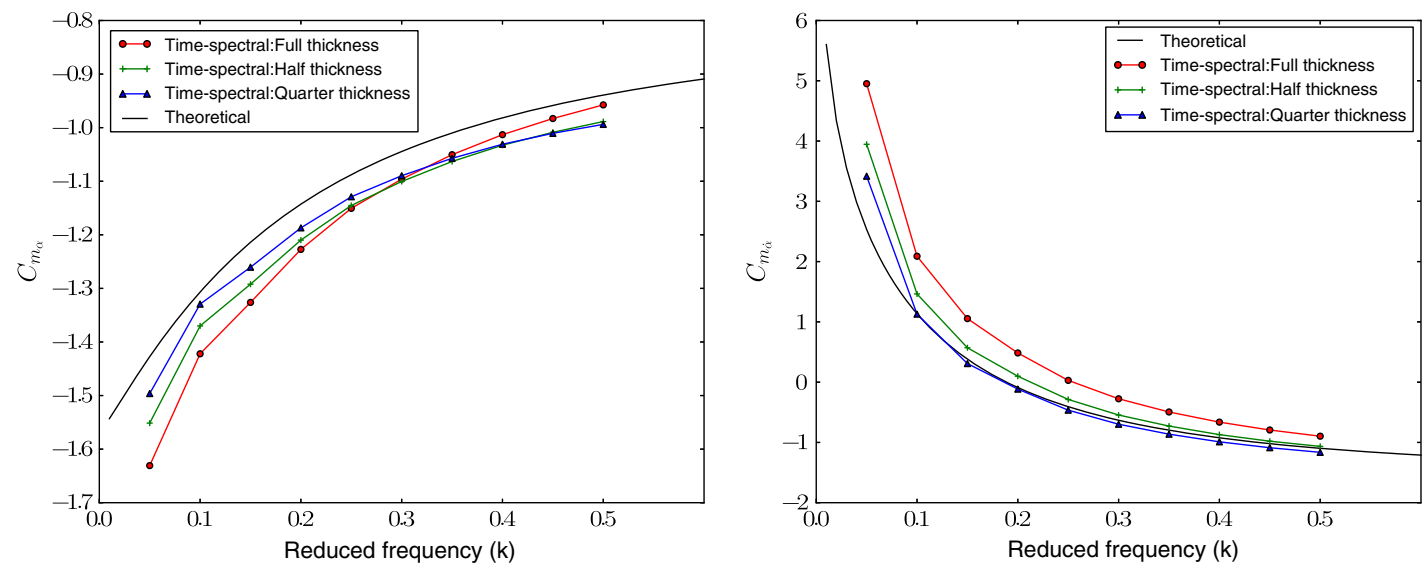

c) $\boldsymbol{C}_{\boldsymbol{m}_{\alpha}}$

d) $\boldsymbol{C}_{\boldsymbol{m}_{\dot{\alpha}}}$

Fig. 5 NACA 0012 time-spectral stability derivative verification, plunging motion at $M=0.1, \alpha=0.00$. 


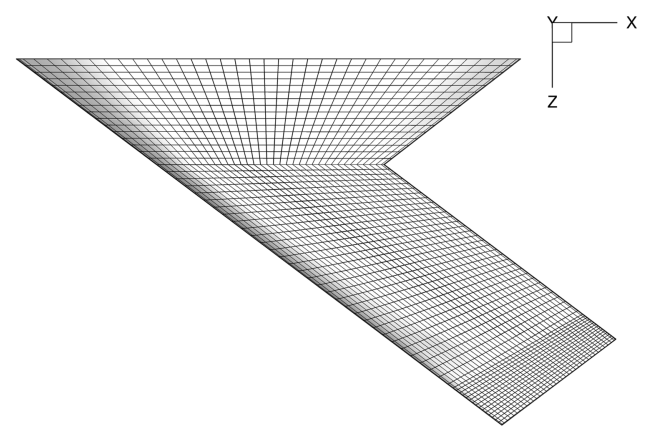

a) Surface mesh

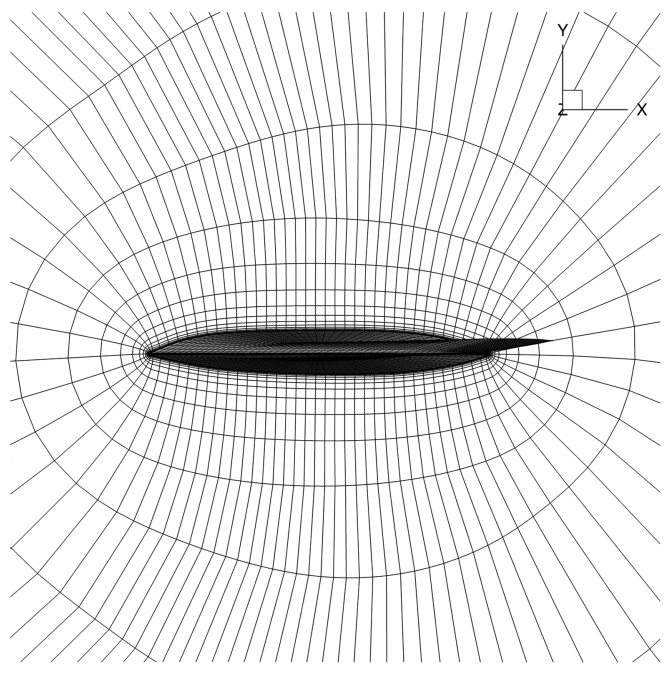

b) Near field mesh at symmetry plane

Fig. 6 SACCON mesh: 185,344 cells.

with a Spalart-Allmaras turbulence model [55]. The surfaces of the wing are modeled as adiabatic viscous walls with a symmetry plane imposed at the root. The off-wall spacing for the 1,482,752 cell mesh is $3 \times 10^{-6} \mathrm{~m}$. Samples of the coarsest mesh are shown in Fig. $\underline{6}$.

Once again, we computed solutions on a series of meshes to assess the numerical accuracy of the solution. We used meshes with $185,344,1,482,752$, and 11,862,016 cells. Assessments of the numerical error associated with $C_{L}$ and its derivatives are shown in Table 3.

The meshes show good mesh convergence as they are refined. The numerical accuracy of $C_{L}$ and $C_{L_{\alpha}}$ is better than $1 \%$ on the finest mesh, whereas the $\dot{\alpha}$ derivative produces an error of $3.5 \%$ on the finest grid. The accuracy of the moment calculations is slightly worse. In this case the error in all three values on the finest mesh is on the order of $2 \%$.

\section{SACCON UCAV: Verification and Validation}

Using the 1,482,752 cell mesh and a RANS flow solver, we ran a series of comparisons with derivatives computed from experimental data by Rohlf et al. [41,56] and CFD results from Le Roy and Morgand [23]. The goal of this comparison is to place the results from the time-spectral computation in context with physical results, thereby validating the method we propose for computing the stability derivatives. The flow conditions used are shown in Table 4. These conditions are selected to match the flow conditions used by Le Roy and Morgand. The results of these comparisons are shown in Figs. 79. Figure 7 shows the lift and moment coefficients for the SACCON configuration compared with both experimental results and steady RANS results from Le Roy and Morgand. Both our CFD results and the reference CFD results differ slightly from the experimental results. The variation in the lift coefficient can be attributed to the effect of the sting from the experimental setup [23]. This effect has been conclusively demonstrated in [23] using simulations with and without the sting. However, our results match the reference CFD results very well. The lift coefficient prediction is essentially the same, whereas there is a slight variation in the moment coefficient.
This variation is likely due to slight variations in the geometry used to generate the test case. As an example, at an angle of attack of 5 deg the norm of the sensitivity of $C_{m}$ with respect to the surface nodes in the mesh is $\mathcal{O}(1)$. At the same angle of attack, the difference between our estimate for $C_{m}$ and the reference is $1.2 \times 10^{-3}$. Therefore, an average change of $1 \mathrm{~mm}$ over the surface of the model would account for the difference. Note also that the steady and time-spectral results for our flow solver coincide, validating the use of the leading coefficient from the linear regression to predict the coefficients.

Figure $\underline{8}$ shows a comparison of the $C_{L}$ derivatives with experimental results from Rohlf et al. $[41,56] . C_{L_{\alpha}}$ matches well, with the time-spectral stability derivative method predicting a value within approximately $2 \%$ of the experimental result at $5 \mathrm{deg}$. The result for $C_{L_{q+\dot{\alpha}}}$ is not as good, with an error of approximately $40 \%$ relative to the experimental results at $5 \mathrm{deg}$. Although this is relatively high, it is on a par with similar CFD results for this configuration, for example those in Rohlf et al. [41,56].

Figure 9 shows a comparison of the $C_{m}$ derivatives with experimental results from Rohlf et al. $[\underline{41}, \underline{56}]$. The $C_{m_{\alpha}}$ derivatives match the experimental results to within approximately $8 \%$ at $5 \mathrm{deg}$. The $C_{m}$ derivatives match surprisingly well, within approximately $10 \%$ at $5 \mathrm{deg}$.

\section{SACCON UCAV: Frequency Dependence}

Because the two-dimensional results show a significant variation with frequency, we conducted a similar frequency comparison for the three-dimensional case. To characterize this effect, we conducted a frequency sweep on the $1,482,752$ cell mesh, the results of which are shown in Fig. 10. Unlike in the two-dimensional case, the $\alpha$ derivatives for the three-dimensional case do tend to a steady-state value as $k \rightarrow 0$. This is consistent with the standard assumptions in linear stability theory. The same cannot be said for the $\dot{\alpha}$ derivatives. Both of these quantities vary continuously as the frequency is varied, indicating that care must be taken when selecting the frequency at which the $\dot{\alpha}$ derivatives are to be evaluated. However, the $\dot{\alpha}$ derivatives seem to vary less significantly over a range from $k=0.03$

Table 3 SACCON mesh refinement results

\begin{tabular}{lllcccc}
\hline \hline Case & \multicolumn{1}{c}{$C_{L}$} & \multicolumn{1}{c}{$C_{m}$} & $C_{L_{\alpha}}$ & $C_{m_{\alpha}}$ & $C_{L_{\dot{\alpha}}}$ & $C_{m_{\dot{\alpha}}}$ \\
\hline 185,000 cell & 0.1036 & 0.01393 & 2.5195 & 0.07998 & -0.4002 & 0.2820 \\
$1,480,000$ cell & 0.1073 & 0.01521 & 2.5932 & 0.1099 & -0.4865 & 0.3021 \\
$11,860,000$ cell & 0.1066 & 0.01607 & 2.5895 & 0.1188 & -0.5459 & 0.3216 \\
Estimate (Richardson extrapolation) & 0.1064 & 0.01635 & 2.5882 & 0.1199 & -0.5656 & 0.3281 \\
$11,860,000$ cell\% error & 0.214 & 1.75 & 0.048 & 2.44 & 3.50 & 1.98 \\
$1,480,000$ cell \% error & 0.859 & 7.0 & .194 & 9.79 & 13.99 & 7.93 \\
\hline \hline
\end{tabular}


Table 4 SACCON test case conditions

\begin{tabular}{lc}
\hline \hline Parameter & Value \\
\hline Mach number & 0.149 \\
Reynolds number & $1.6 \times 10^{6}$ \\
$\alpha$ (deg) & $0-10$ \\
Half-body reference area (m) & 0.385 \\
Reference half-span (m) & 0.769 \\
Reference chord (m) & 0.479 \\
Frequency (Hz) & 1 \\
Reference moment center (m) & 0.6 \\
Reference rotational center (m) & 0.855414 \\
\hline \hline
\end{tabular}

to 0.3 , making that a reasonable range of frequencies to use for this case. Note that the simulations and experiments in the previous section were both computed at the same frequency of $1 \mathrm{~Hz}$, and so the comparisons made there are still valid.

\section{Optimization Problem}

To demonstrate the use of the preceding stability derivatives in an optimization problem, we consider a series of Euler drag minimization problems for an untapered wing. In each optimization we add a single stability derivative constraint to quantify its effect on the optimal solution. The details of the optimizations are described next. A much more detailed optimization study demonstrating the effects of various stability constraints on flying wings has been conducted by the authors using the methods outlined in this paper. In that study, the authors used the method presented here to demonstrate that at subsonic and low transonic Mach numbers airfoil shape modifica-

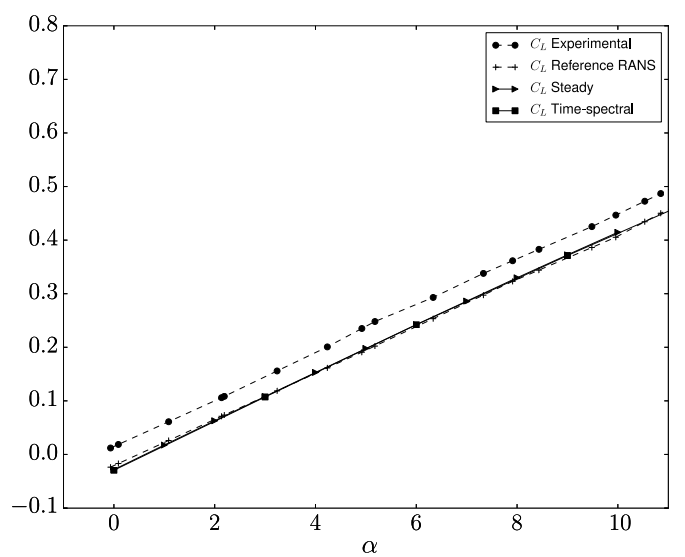

a) $C_{L}$ tion was sufficient to ensure statically stable trimmed flight with essentially no drag penalty, whereas at higher Mach numbers sweep and twist were also required to ensure efficient stable flight. Further, the authors showed that to satisfy a handling-quality constraint, sweep and twist were required at all speeds. This study was made possible by the method for computing the gradient of the transient derivatives presented herein.

\section{A. Test Case}

The wing used for the current study is a straight rectangular wing with a NACA 0012 airfoil profile as described by Hicken and Zingg [57]. The wing has a half-span of $3 \mathrm{~m}$ and a chord of $1 \mathrm{~m}$, giving it an aspect ratio of six. It has a taper ratio of one and a leading-edge sweep angle of 0 deg. The details of the geometry are summarized in Table 5. The three optimization results shown are computed on a mesh with $1,105,920$ cells. The mesh has a C-O topology and is split into 32 blocks, with the surface mesh having 97 points spanwise and 81 points from the leading edge to the trailing edge on each of the top and bottom surfaces of the wing. The far-field boundary is approximately 15 chords from the wing, and the off-wall spacing of the mesh is $1 \times 10^{-3} \mathrm{~m}$ at the leading edge and $5 \times 10^{-4} \mathrm{~m}$ at the trailing edge. The flow solutions are computed with an Euler flow solver and are analyzed at $M=0.78$.

\section{B. Mesh Convergence}

To demonstrate the accuracy of the mesh used, we performed a mesh convergence study, the results of which are shown in Table 6 . As the table shows, the mesh is sufficiently refined, with errors of less than $1 \%$ for $C_{L}, C_{D}$, and $C_{L_{\alpha}}$ and an error of $1.1 \%$ for $C_{m_{\alpha}}$.

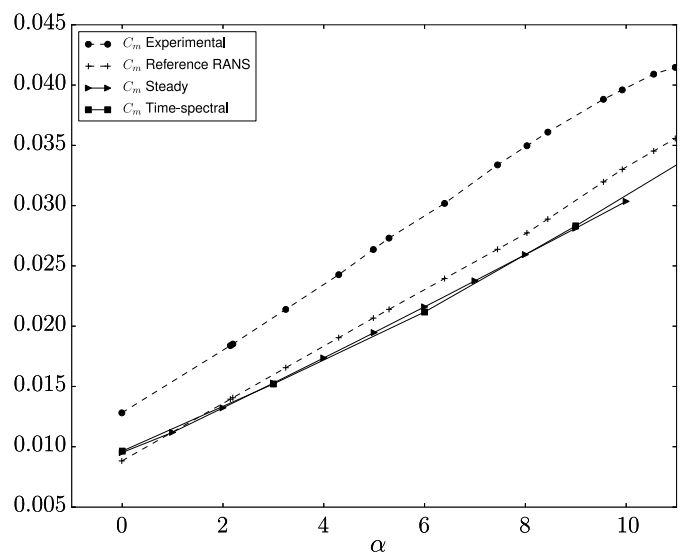

b) $C_{m}$

Fig. 7 Comparison of $C_{L}$ and $C_{m}$ for the SACCON UCAV with experimental and RANS results [23].

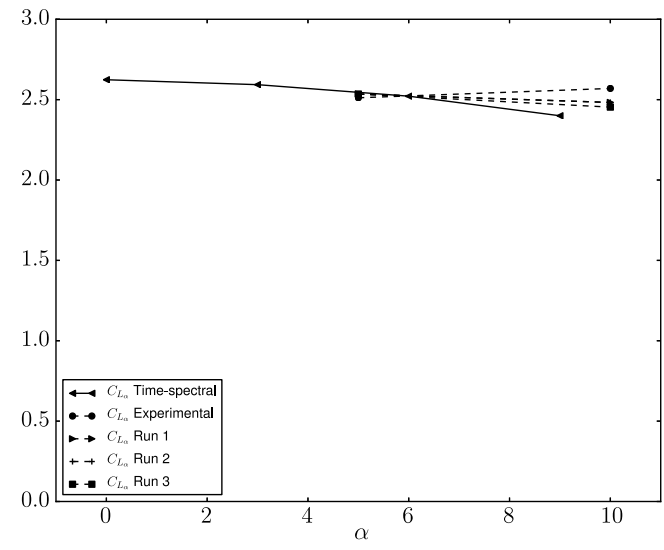

a) $C_{L_{\alpha}}$ derivatives

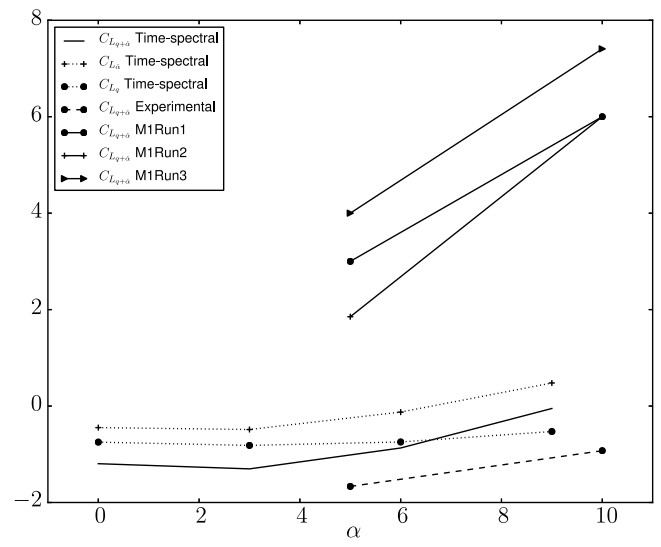

b) $C_{L_{q+\dot{\alpha}}}$ derivatives

Fig. 8 Comparison of SACCON UCAV $C_{L}$ derivatives with experimental results [41]. 


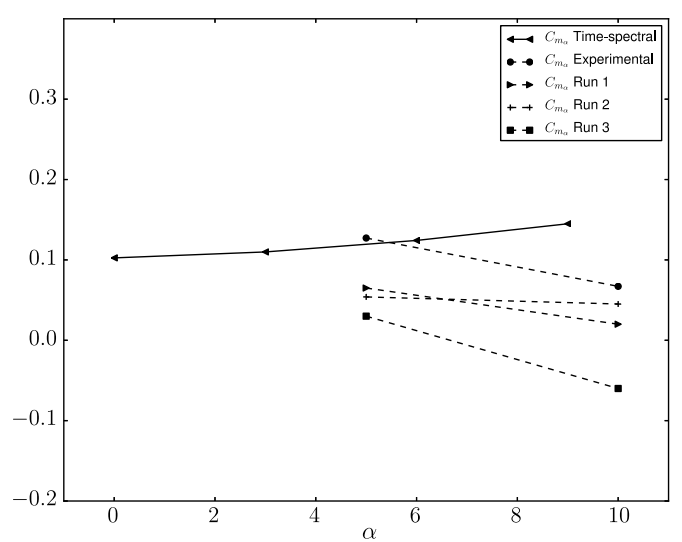

a) $C_{m_{\alpha}}$ derivatives

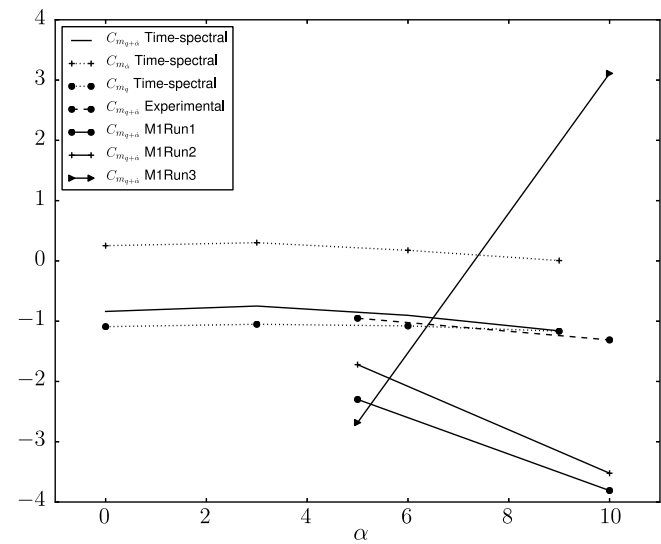

b) $C_{m_{q+\alpha}}$ derivatives

Fig. 9 Comparison of SACCON UCAV $C_{m}$ derivatives with experimental results [41].

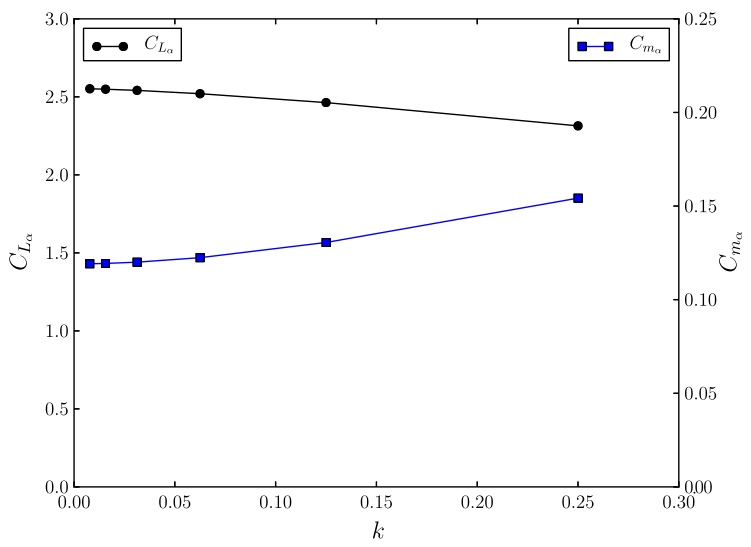

a) $\alpha$ derivatives

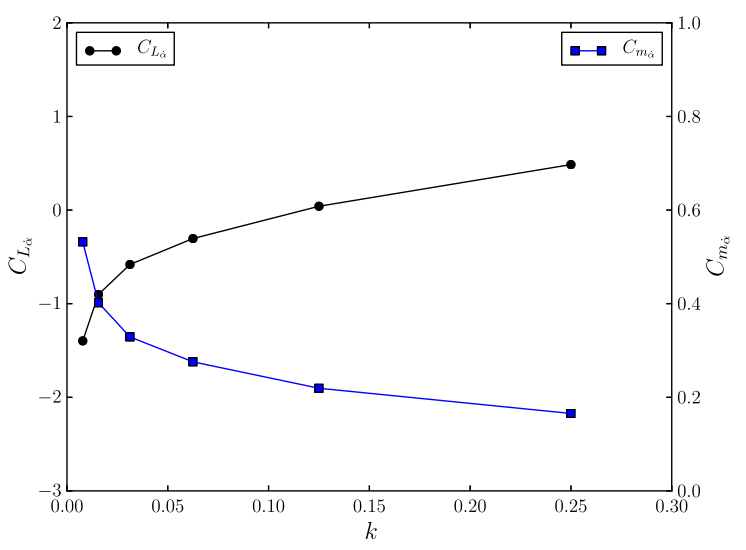

b) $\dot{\alpha}$ derivatives

Fig. 10 Frequency dependence of the derivatives for the SACCON UCAV.

\section{Problem Description}

The formulation of the optimization problem is as follows:

minimize $C_{D}$

w.r.t. $\alpha, \theta_{i}, b, c, \lambda, \Delta \mathbf{y}_{\mathrm{FFD}}, x_{\mathrm{CG}}$

subject to $C_{L_{\text {ref }}}-C_{L} \leq 0$

$$
C_{m}=0
$$

$C_{b}-C_{b_{\text {ref }}}=0$

$A=A_{\text {ref }}$

$C_{m_{\alpha}} \leq-0.5$ (Case 2 only)

$2.0 \leq C_{m_{\dot{\alpha}}} \leq 2.01$ (Case 3 only)

This optimization problem is a lift-constrained drag minimization with a trim constraint. Because the optimization is based on an Euler CFD solver, there is no significant penalty for making the wing area larger. Therefore, we have constrained $C_{L}$ rather than lift, and we have constrained the area of the wing to keep the overall lift

Table 5 Baseline wing: geometry specifications

\begin{tabular}{lc}
\hline \hline Parameter & Value \\
\hline Half-wing area $(\mathrm{m})$ & 3.0 \\
Half-wing span $(\mathrm{m})$ & 3.0 \\
Chord $(\mathrm{m})$ & 1.0 \\
Leading-edge sweep $(\mathrm{deg})$ & 0.0 \\
Taper ratio & 1.0 \\
Wing tip washout (deg) & 0.0 \\
Wing dihedral & 0.0 \\
\hline \hline
\end{tabular}

provided by the wing constant. We have also added a root bending moment constraint. This constraint considers both the spanwise and streamwise extent of the wing, computing a bending moment about an effective axis that stays roughly aligned with the major axis of the wing as the sweep is increased. This constraint penalizes both increased span and increased sweep, forcing the optimizer to consider the tradeoff between sweep and span, and consequently wave drag and induced drag, in the transonic regime. Full details of the implementation of this constraint are presented in Mader and Martins [5].

The optimizer is able to vary the section shape $\left(\Delta \mathbf{y}_{\mathrm{FFD}}\right)$, twist $\left(\theta_{i}\right)$, and planform $(b, c, \lambda)$ of the wing to minimize the drag. The value of the bending coefficient at the root is constrained to be that of an elliptical lift distribution at $M=0.5$ for the same lift. Using this basic formulation, we have conducted three variants of this optimization. The first optimization is conducted exactly as described previously to provide a reference case for the study. In the second optimization, we constrain $C_{m_{\alpha}}$ to be less than or equal to -0.5 . This ensures that the design is statically stable at the optimum. In the third optimization,

Table 6 Baseline wing: mesh refinement results

\begin{tabular}{lcccc}
\hline \hline Case & $C_{L}$ & $C_{D}$ & $C_{L_{\alpha}}$ & $C_{m_{\text {。 }}}$ \\
\hline 138,000 cell & 0.262161 & 0.004065 & 5.00924 & 1.19912 \\
$1,100,000$ cell & 0.261608 & 0.003787 & 4.99286 & 1.18342 \\
$8,800,000$ cell & 0.261322 & 0.003769 & 4.98115 & 1.17374 \\
Estimate (Richardson & 0.261228 & 0.003763 & 4.97724 & 1.17051 \\
extrapolation) & & & & \\
$1,100,000$ cell \% error & 0.146 & 0.621 & 0.314 & 1.10 \\
\hline \hline
\end{tabular}




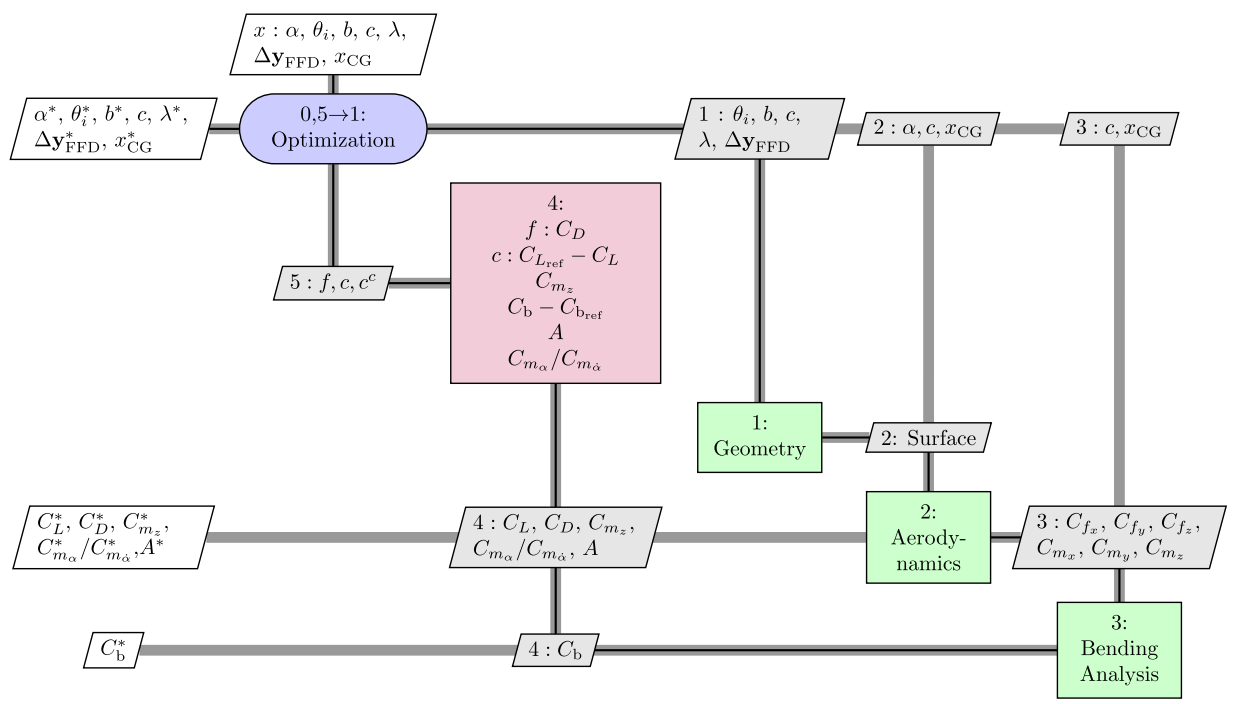

Fig. 11 XDSM for the optimization problems.

we constrain $C_{m_{\dot{\alpha}}}$ to be between 2 and 2.01. The value of this constraint was selected to differentiate the results of this optimization from the other two cases and to demonstate that the value of $C_{m_{\dot{\alpha}}}$ could be used as an independent constraint. These last two optimizations demonstrate the use of the time-spectral stability derivatives in an optimization context. Figure 11 depicts the data and process flow for these problems in the extended design structure matrix (XDSM) format of Lambe and Martins [58]. In this class of diagrams, the diagonal blocks represent the disciplines, whereas the offdiagonal blocks and light gray lines represent the data flow between the disciplines. The thin black line represents the process flow through the optimization problem.

\section{Design Variables}

The design variables used in our optimization problem include planform variables, such as sweep and span, as well as 280 surfaceshape design variables. The surface-shape design variables, the control points of an Free-form deformation (FFD) volume, modify the surface shape of the wing and affect both its streamwise and spanwise profile. Table 7 lists the design variables and their respective bounds.

\section{Optimization Results}

The results of the three optimization cases are shown in Figs. 1214. Each figure shows the $C_{p}$ distribution on the top surface of the airfoil as well as the spanwise lift distribution, the spanwise twist distribution, and the section shape and $C_{p}$ at three spanwise stations. In the reference problem shown in Fig. 12 we see that the optimizer has modified the section shape of the wing to eliminate the peaky section profile typical of the original NACA 0012 sections. The sections of the optimized wing all produce rooftop $C_{p}$ profiles, which reduces the wave drag. Unfortunately, although this change in pressure distribution is advantageous from a drag reduction perspective, it has also made the wing unstable, because the CG location required to trim the aircraft is now aft of the neutral point. Note that the lift distribution is also skewed slightly inboard relative

Table 7 Design variables and their bounds

\begin{tabular}{lcrr}
\hline \hline Design variable & Symbol & Lower bound & Upper bound \\
\hline Angle of attack (deg) & $\alpha$ & -15 & 15 \\
Section twist at nine sections (deg) & $\theta_{i}$ & -10 & 10 \\
Span (m) & $b$ & 2.0 & 3.2 \\
Chord (m) & $c$ & 0.5 & 1.4 \\
Sweep (deg) & $\lambda$ & 0 & 45.0 \\
CG location & $x_{\mathrm{CG}}$ & -20 & 20 \\
FFD control points: $y$ offset (m) & $\Delta \mathbf{y}_{\mathrm{FFD}}$ & -0.075 & 0.075 \\
\hline \hline
\end{tabular}

to an elliptic distribution, which allows for an extension of the span while maintaining the required root bending moment, a typical aerostructural tradeoff. In the $C_{m_{\alpha}}$ constrained optimization shown in Fig. 13 the optimizer has changed two main characteristics of the wing to satisfy the constraint: It has increased the sweep and added reflex to the trailing edge of the airfoil. The addition of sweep allows the section profiles to have a slightly higher critical $C_{p}$, giving the optimizer more freedom to alter the section shapes. It also shifts the neutral point of the wing aft, which helps to stabilize the aircraft. The addition of trailing-edge reflex to the section shapes of the wing shifts the $\mathrm{CG}$ location required to trim the aircraft forward and helps to directly satisfy the $C_{m_{\alpha}}$ constraint. Note that in this case the CG location to trim the aircraft has shifted forward of the neutral point, indicating a positive static margin, or negative $C_{m_{\alpha}}$, as required by the problem formulation. Note also that the coordinate system used in the CFD computations provides pitching moments that are opposite to the generally accepted sign convention in the stability and control community, and so we have multiplied all of our results by -1 so that the values presented match the standard convention.

The third optimization, the $C_{m_{\dot{\alpha}}}$ constrained case, produces a shape that is a mixture of the results from the previous two cases. As in the reference case, the root section has a rooftop profile with no trailingedge reflex, whereas the tip section has significant trailing-edge reflex, similarly to the second case. Note that the CG location to trim and the neutral point are essentially coincident, producing a neutrally stable aircraft.

Finally, looking at the results shown in Table 8 we can see that the three optimal solutions produce drag coefficients within one count of each other. This indicates that under the conditions modeled no significant penalty is paid to satisfy the various stability constraints. Further, in the second and third cases, respectively, the $C_{m_{\alpha}}$ and $C_{m_{\dot{\alpha}}}$ constraints are satisfied exactly to the tolerance specified. This demonstrates that the proposed method for computing stability derivatives and including them in gradient-based optimizations is valid.

\section{Computational Performance}

The optimizations conducted in this work were run on 80 Intel Nehalem Xeon E5540 processors (eight cores, 16 GB ram per node) with a mix of DDR and QDR InfiniBand interconnections. The time-spectral flow solutions took approximately $300 \mathrm{~s}$ to compute, whereas the individual time-spectral adjoint solutions averaged approximately $370 \mathrm{~s}, 123 \%$ of the solution time, and assembling the Jacobian took approximately $110 \mathrm{~s}$. This leads to a total optimization cycle time (one solution plus four adjoint solutions for the reference case) of approximately $1800 \mathrm{~s}$ or $30 \mathrm{~min}$. The cycle time for the second two optimizations is slightly longer because they each have one extra adjoint to solve (the adjoint of the stability derivative). The 


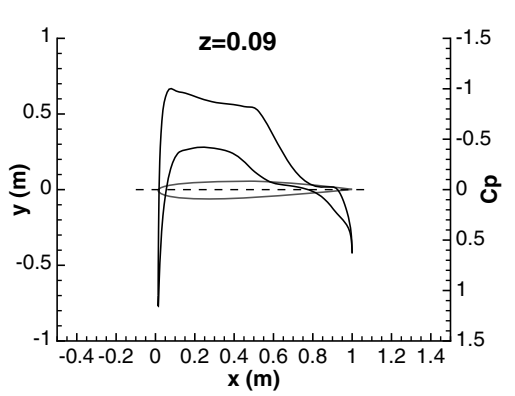

○ CG

- Neutral Point

Cp: $\quad-1 \quad-0.6 \quad-0.2 \quad 0.2 \quad 0.6 \quad 1$
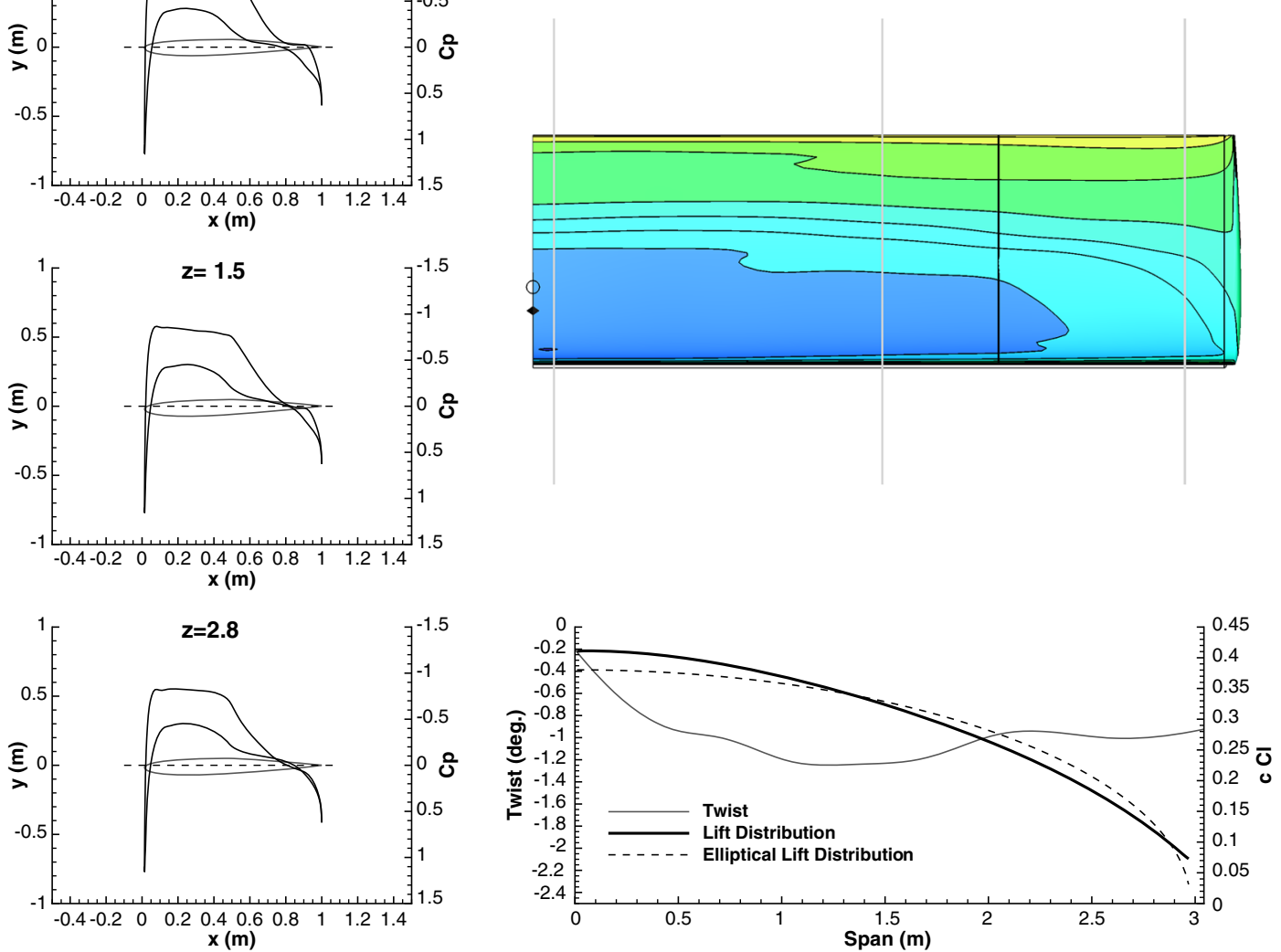

Fig. 12 Reference problem: $M=0.78, C_{L}=0.3, C_{m}=0$.

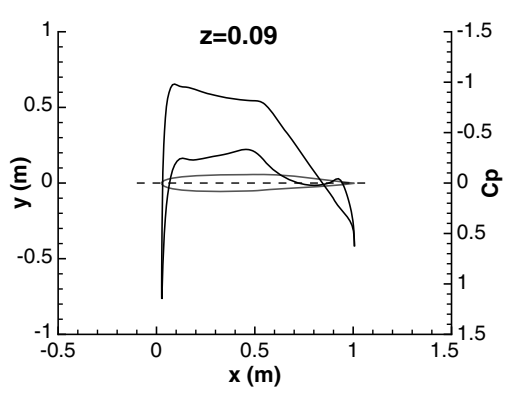

O $\mathbf{C G}$

- Neutral Point

Cp: $\quad-1 \quad-0.6 \quad-0.20 .2 \quad 0.6 \quad 1$
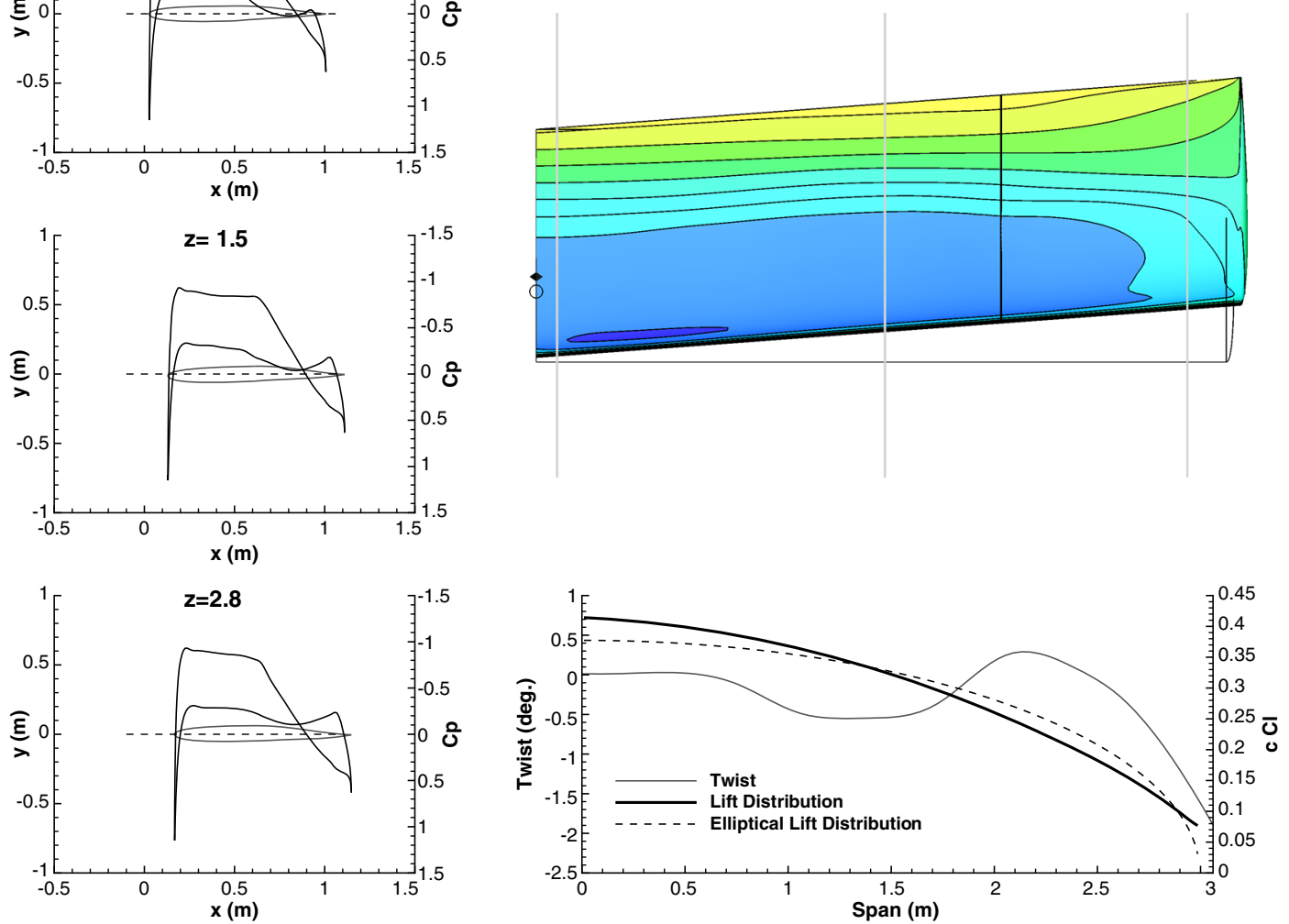

Fig. $13 C_{m_{\alpha}}$ problem: $M=0.78, C_{L}=0.3, C_{m}=0$. 


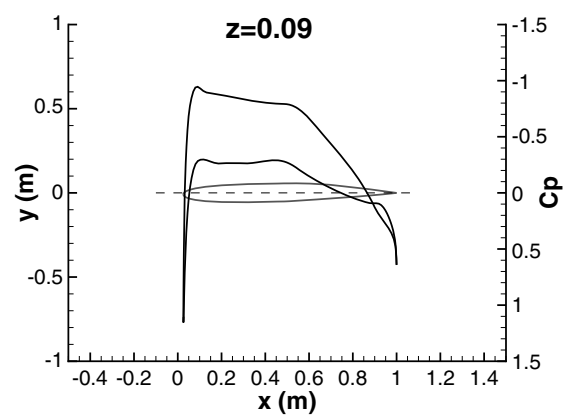

O CG

- Neutral Point

$\mathrm{Cp}:$
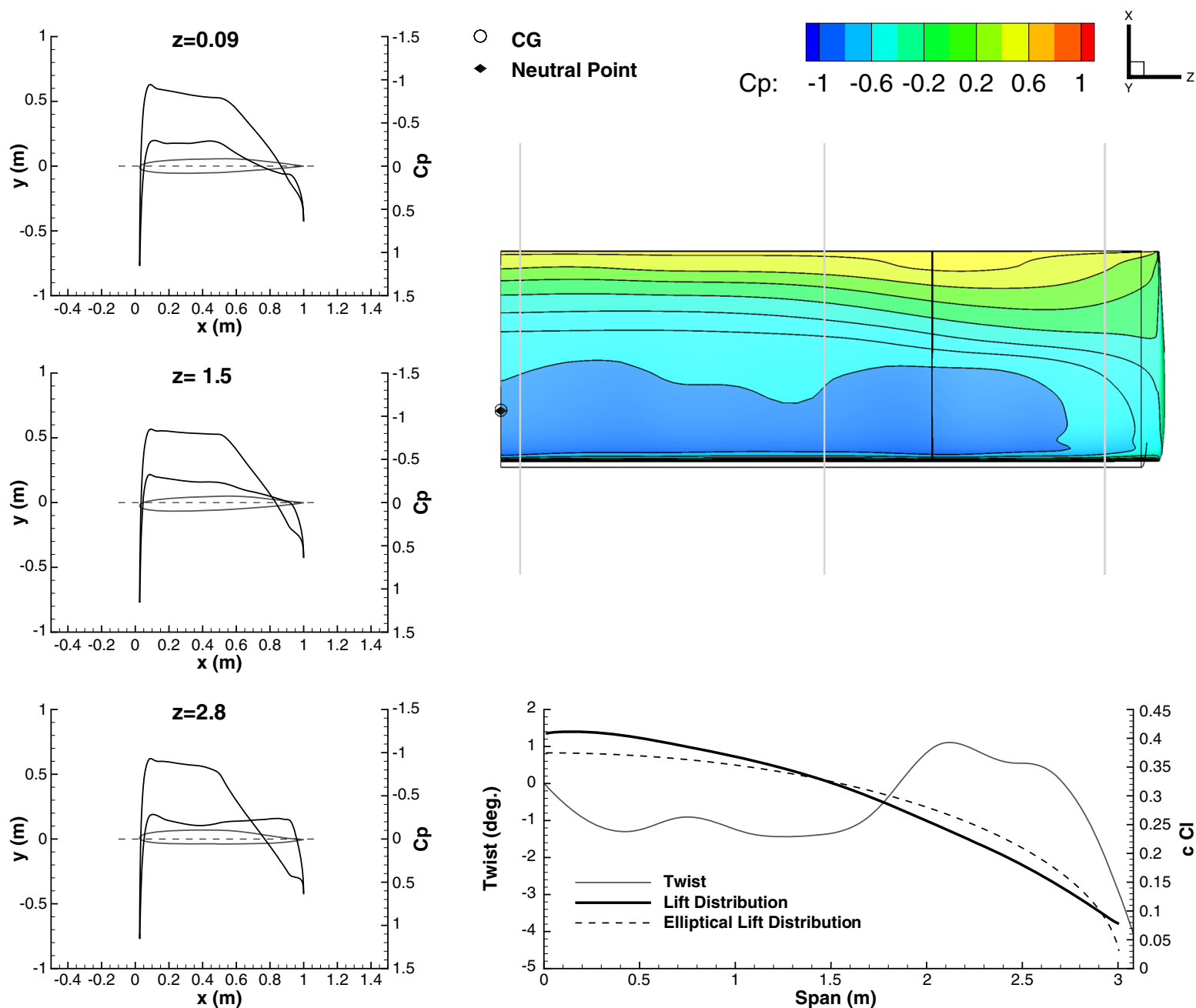

Fig. $14 C_{m_{\dot{\alpha}}}$ problem: $M=0.78, C_{L}=0.3, C_{m}=0$.

complete reference optimization took just over a day of wall time, the $C_{m_{\alpha}}$ case took two and a half days of wall time, and the $C_{m_{\dot{\alpha}}}$ case took just over four days of wall time. Although these computational times are significant, they are certainly reasonable for this level of optimization. Figure 15 shows the convergence history for the optimality criterion of each case. All three initially converge quite rapidly, with the convergence slowing somewhat as the optimality reaches an order of $10^{-4}$. The two stability-constrained cases take more iterations to converge than the reference case. This is expected given the more complex nature of the physical tradeoffs inherent in these problem formulations. However, in the $C_{m_{\dot{\alpha}}}$ case, the optimizer completes several optimization iterations with the optimality tolerance below the required level. This occurs because the optimizer is alternating between satisfying the feasibility and the optimality of the problem. It is possible that with some modifications to the optimizer options this performance could be improved.

Table 8 Optimization results: $M=0.78, C_{L}=0.3, C_{m}=0$

\begin{tabular}{lccc}
\hline \hline Parameter & Baseline & $C_{m_{\bullet}}$ & $C_{m_{\dot{*}}}$ \\
\hline$C_{D}$ & 0.00515 & 0.00516 & 0.00505 \\
$C_{m_{\alpha}}$ & 0.75661 & -0.50000 & 0.01935 \\
$C_{m_{\dot{\alpha}}}$ & 5.14469 & -0.06137 & 2.01000 \\
$\alpha(\mathrm{deg})$ & 2.97 & 2.99 & 2.98 \\
$\lambda(\mathrm{deg})$ & 0.00 & 4.23 & 0.000 \\
$b(\mathrm{~m})$ & 3.07 & 3.09 & 3.11 \\
$c(\mathrm{~m})$ & 0.986 & 0.980 & 0.974 \\
\hline \hline
\end{tabular}

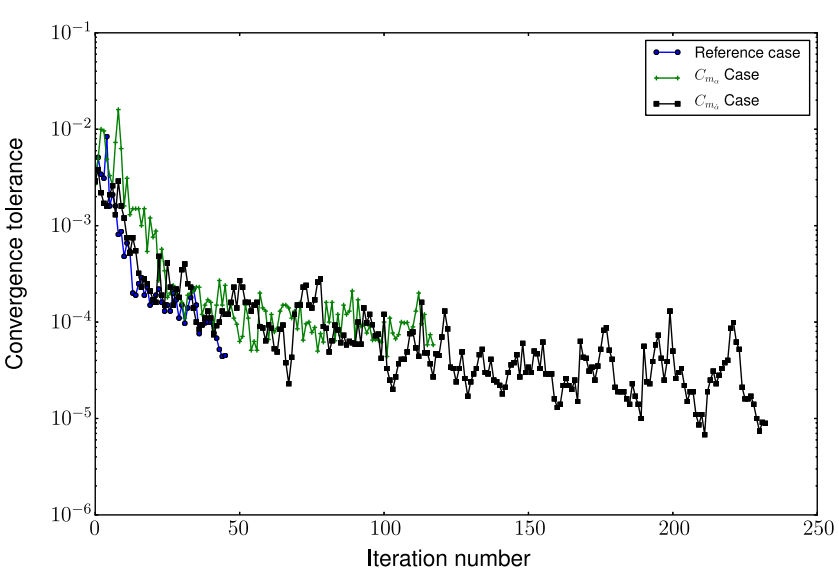

Fig. 15 Optimality convergence history.

\section{Conclusions}

An approach to computing aircraft stability derivatives for use in CFD-based aerodynamic shape optimization has been introduced, verified, and validated using an Euler and RANS CFD solver. Two-dimensional Euler CFD results have been presented, verifying the approach against a theoretical plunging airfoil case. Threedimensional RANS CFD results have been presented in which the approach was able to compute a $C_{L_{\alpha}}$ derivative within $10 \%$ of the experimental result for the SACCON UCAV configuration, confirming the validity of the approach. Further, a small set of Euler CFD-based optimizations has been conducted, demonstrating the usefulness of 
the approach for gradient-based aerodynamic shape optimization. In particular, the approach has been used to constrain the values of $C_{m_{\alpha}}$ and $C_{m_{\dot{m}}}$ to a numerical accuracy of $10^{-5}$ in a drag minimization problem, thereby demonstrating the effectiveness of the approach.

\section{Acknowledgments}

The authors are grateful for the funding provided by the Canada Research Chair program and the Natural Sciences and Engineering Research Council. The computations were performed on the General Purpose Cluster (GPC) supercomputer at the SciNet Highperformance computing (HPC) Consortium. SciNet is funded by the Canada Foundation for Innovation under the auspices of Compute Canada; the Government of Ontario; the Ontario Research Fund, Research Excellence; and the University of Toronto. In addition, we would like to thank Edwin van der Weide for his assistance in the early stages of this project, in particular with the time-spectral formulation in the SUmb flow solver, as well as Gaetan Kenway for his assistance in generating RANS meshes for the SACCON case.

\section{References}

[1] Jameson, A., "Aerodynamic Design via Control Theory," Journal of Scientific Computing, Vol. 3, No. 3, 1988, pp. 233-260. doi:10.1007/BF01061285

[2] Hicken, J., and Zingg, D. W., "Aerodynamic Optimization Algorithm with Integrated Geometry Parameterization and Mesh Movement," AIAA Journal, Vol. 48, No. 2, 2010, pp. 400-413. doi: $10.2514 / 1.44033$

[3] Reuther, J. J., Jameson, A., Alonso, J. J., Rimlinger, M. J., and Saunders, D., "Constrained Multipoint Aerodynamic Shape Optimization Using an Adjoint Formulation and Parallel Computers, Part 1," Journal of Aircraft, Vol. 36, No. 1, 1999, pp. 51-60. doi: $10.2514 / 2.2413$

[4] Reuther, J. J., Jameson, A., Alonso, J. J., Rimlinger, M. J., and Saunders, D., "Constrained Multipoint Aerodynamic Shape Optimization Using an Adjoint Formulation and Parallel Computers, Part 2," Journal of Aircraft, Vol. 36, No. 1, 1999, pp. 61-74. doi: $10.2514 / 2.2414$

[5] Mader, C. A., and Martins, J. R. R. A., "Stability-Constrained Aerodynamic Shape Optimization of Flying Wings," Journal of Aircraft, Vol. 50, No. 5, 2013, pp. 1431-1449. doi:10.2514/1.C031956

[6] Lyu, Z., and Martins, J. R. R. A., "Aerodynamic Shape Optimization of a Blended-Wing-Body Aircraft Using Navier-Stokes Equations," Journal of Aircraft (to be published). doi:10.2514/1.C032491

[7] Kenway, G. K. W., and Martins, J. R. R. A., "Multi-Point High-Fidelity Aerostructural Optimization of a Transport Aircraft Configuration," Journal of Aircraft, Vol. 51, No. 1, 2014, pp. 144-160. doi:10.2514/1.C032150

[8] Kenway, G. K. W., Kennedy, G. J., and Martins, J. R. R. A., "A Scalable Parallel Approach for High-Fidelity Steady-State Aeroelastic Analysis and Derivative Computations," AIAA Journal (in press). doi:10.2514/1.J052255

[9] Charlton, E. F., "Numerical Stability and Control Analysis Towards Falling-Leaf Prediction Capabilities of SPLITFLOW for Two Generic High-Performance Aircraft Models," NASA CR-1998-208730, 1998.

[10] Godfrey, A. G., and Cliff, E. M., "Direct Calculation of Aerodynamic Force Derivatives: A Sensitivity-Equation Approach," AIAA Paper 1998-0393, 1998.

[11] Park, M. A., Green, L. L., Montgomery, R. C., and Raney, D. L., "Determination of Stability and Control Derivatives Using Computational Fluid Dynamics and Automatic Differentiation," AIAA Paper 1999-3136, 1999.

[12] Bischof, C., Carle, A., Corliss, G., Grienwank, A., and Hoveland, P., "ADIFOR: Generating Derivative Codes from Fortran Programs," Scientific Programming, Vol. 1, No. 1, 1992, pp. 11-29.

[13] Park, M. A., and Green, L. L., "Steady-State Computation of Constant Rotational Rate Dynamic Stability Derivatives," AIAA Paper 20004321, 2000.

[14] Limache, A., and Cliff, E., "Aerodynamic Sensitivity Theory for Rotary Stability Derivatives," Journal of Aircraft, Vol 37, No. 4, 2000, pp. 676-683. doi: $10.2514 / 2.2651$
[15] Babcock, D. A., and Arena, A. S., "Estimating Aircraft Stability Derivatives Through Finite Element Analysis," AIAA Paper 20045174, 2004.

[16] Mader, C. A., and Martins, J. R. R. A., "Computation of Aircraft Stability Derivatives Using an Automatic Differentiation Adjoint Approach," AIAA Journal, Vol. 49, No. 12, 2011, pp. 2737-2750. doi:10.2514/1.J051147

[17] Weinacht, P., "Navier-Stokes Predictions of the Individual Components of the Pitch-Damping Sum,' Journal of Spacecraft and Rockets, Vol. 35, No. 5, 1998, pp. 598-605 doi: $10.2514 / 2.3390$

[18] DeSpirito, J., Silton, S. I., and Weinacht, P., "Navier-Stokes Predictions of Dynamic Stability Derivatives: Evaluation of Steady-State Methods," Army Research Laboratory TR-4605, 2008.

[19] Sahu, J., "Numerical Computations of Dynamic Derivatives of a Finned Projectile Using a Time-Accurate CFD Method," AIAA Paper 20076581, 2007.

[20] Oktay, E., and Akay, H. U., "CFD Predictions of Dynamic Derivatives for Missiles," AIAA Paper 2002-0276, 2002.

[21] Murman, S. M., "Reduced-Frequency Approach for Calculating Dynamic Derivatives," AIAA Journal, Vol. 45, No. 6, 2007, pp. 1161-1168. doi:10.2514/1.15758

[22] Mialon, B., Khrabov, A., Da Ronch, A., Cavagna, L., Zhang, M., and Ricci, S., "Benchmarking the Prediction of Dynamic Derivatives: Wind Tunnel Tests, Validation, Acceleration Methods," AIAA Paper 2010$8244,2010$.

[23] Le Roy, J.-F., and Morgand, S., "SACCON CFD Static and Dynamic Derivatives Using elsA," AIAA Paper 2010-4562, 2010.

[24] Da Ronch, A., Vallespin, D., Ghoreyshi, M., and Badcock, K., "Computation of Dynamic Derivatives Using CFD," AIAA Paper 20104817, 2010.

[25] Cummings, R. M., Jirásek, A., Petterson, K., and Schmidt, S., "SACCON Static and Dynamic Motion Flow Physics Simulation Using Cobalt," AIAA Paper 2010-4691, 2010.

[26] Tormalm, M., and Schmidt, S., "Computational Study of Static and Dynamic Vortical Flow over the Delta Wing SACCON Configuration Using the FOI Flow Solver Edge," AIAA Paper 2010-4561, 2010.

[27] Da Ronch, A., Ghoreyshi, M., Badcock, K., Görtz, S., Widhalm, M., Dwight, R., and Campobasso, M., "Linear Frequency Domain and Harmonic Balance Predictions of Dynamic Derivatives," AIAA Paper 2010-4699, 2010.

[28] Da Ronch, A., Vallespin, D., Ghoreyshi, M., and Badcock, K. J., "Evaluation of Dynamic Derivatives Using Computational Fluid Dynamics," AIAA Journal, Vol. 50, No. 2, 2012, pp. 470-484. doi:10.2514/1.J051304

[29] Da Ronch, A., McCracken, A. J., and Badcock, K. J., "Linear Frequency Domain and Harmonic Balance Predictions of Dynamic Derivatives," Journal of Aircraft, Vol. 50, No. 3, 2013, pp. 694-707. doi:10.2514/1.C031674

[30] Ghate, D. P., and Giles, M. B., "Efficient Hessian Calculation Using Automatic Differentiation," AIAA Paper 2007-4059, 2007.

[31] Rumpfkeil, M. P., and Mavriplis, D. J., "Efficient Hessian Calculations Using Automatic Differentiation and the Adjoint Method with Applications," AIAA Journal, Vol. 48, No. 10, 2010, pp. 2406-2417. doi:10.2514/1.J050451

[32] Liem, R. P., Mader, C. A., Lee, E., and Martins, J. R. R. A., "Aerostructural Design Optimization of a 100-Passenger Regional Jet with Surrogate-Based Mission Analysis," AIAA Paper 2013-2414, 2013.

[33] Nadarajah, S. K., and Jameson, A., "Optimum Shape Design for Un steady Flows with Time-Accurate Continuous and Discrete Adjoint Methods," AIAA Journal, Vol. 45, No. 7, 2007, pp. 1478-1491. doi: $10.2514 / 1.24332$

[34] Mani, K., and Mavriplis, D. J., "Unsteady Discrete Adjoint Formulation for Two-Dimensional Flow Problems with Deforming Meshes," AIAA Journal, Vol. 46, No. 6, 2008, pp. 1351-1364. doi: $10.2514 / 1.29924$

[35] Rumpfkeil, M. P., and Zingg, D. W., "The Optimal Control of Unsteady Flows with a Discrete Adjoint Method," Optimization and Engineering, Vol. 11, No. 1, 2010, pp. 5-22. doi:10.1007/s11081-008-9035-5

[36] Mavriplis, D. J., "Solution of the Unsteady Discrete Adjoint for ThreeDimensional Problems on Dynamically Deforming Unstructured Meshes," AIAA Paper 2008-727, 2008.

[37] Nielsen, E. J., Diskin, B., and Yamaleev, N. K., "Discrete AdjointBased Design Optimization of Unsteady Turbulent Flows on Dynamic Unstructured Grids," AIAA Journal, Vol. 48, No. 6, 2010, pp. 1195-1206.

doi: $10.2514 / 1 . J 050035$ 
[38] Nadarajah, S. K., and Jameson, A., "Optimum Shape Design for Unsteady Three-Dimensional Viscous Flows Using a Nonlinear Frequency-Domain Method," Journal of Aircraft, Vol. 44, No. 5, 2007, pp. 1513-1527. doi:10.2514/1.27601

[39] Choi, S., Potsdam, M., Lee, K., Iaccarino, G., and Alonso, J. J., "Helicopter Rotor Design Using a Time-Spectral and Adjoint-Based Method," AIAA Paper 2008-5810, 2008.

[40] Etkin, B., Dynamics of Atmospheric Flight, Dover, Mineola, NY, 2000, pp. 158-165, 276-285.

[41] Rohlf, D., Schmidt, S., and Irving, J., "Stability and Control Analysis for an Unmanned Aircraft Configuration Using System-Identification Techniques," Journal of Aircraft, Vol. 49, No. 6, 2012, pp. 1597-1609. doi:10.2514/1.C031392

[42] Hall, K. C., Thomas, J. P., and Clark, W. S., "Computation of Unsteady Nonlinear Flows in Cascades Using a Harmonic Balance Technique," AIAA Journal, Vol. 40, No. 5, 2002, pp. 879-886.

[43] Ekici, K., and Hall, K. C., "Nonlinear Analysis of Unsteady Flows in Multistage Turbomachines Using Harmonic Balance," AIAA Journal, Vol. 45, No. 5, 2007, pp. 1047-1057. doi:10.2514/1.22888

[44] McMullen, M., and Jameson, A., "The Computational Efficiency of Non-Linear Frequency Domain Methods," Journal of Computational Physics, Vol. 212, No. 2, 2006, pp. 637-661. doi:10.1016/j.jcp.2005.07.021

[45] Mc Mullen, M., Jameson, A., and Alonso, J., "Demonstration of Nonlinear Frequency Domain Methods," AIAA Journal, Vol. 44, No. 7, 2006, pp. 1428-1435. doi:10.2514/1.15127

[46] Gopinath, A. K., and Jameson, A., "Time Spectral Method for Periodic Unsteady Computations over Two- and Three-Dimensional Bodies," AIAA Paper 2005-1220, 2005.

[47] Van der Weide, E., Kalitzin, G., Schluter, J., and Alonso, J. J., "Unsteady Turbomachinery Computations Using Massively Parallel Platforms," AIAA Paper 2006-0421, 2006.

[48] Van der Weide, E., Gopinath, A. K., and Jameson, A., "Turbomachinery Applications with the Time Spectral Method," AIAA Paper 2005-4905, 2005.
[49] Lyu, Z., Kenway, G. K., Paige, C., and Martins, J. R. R. A., "Automatic Differentiation Adjoint of the Reynolds-averaged Navier-Stokes Equations with a Turbulence Model," 21st AIAA Computational Fluid Dynamics Conference, AIAA Paper 2013-2581, 2013.

[50] Gopinath, A. K., Efficient Fourier-Based Algorithms for Time-Periodic Unsteady Problems, Ph.D. Thesis, Stanford Univ., Stanford, CA, April 2007.

[51] Qin, N., Ludlow, D., Shaw, S., Edwards, J., and Dupuis, A., "Calculation of Pitch Damping Coefficients for Projectiles," AIAA Paper 1997-0405, 1997.

[52] Mader, C. A., and Martins, J. R. R. A., "Derivatives for Time-Spectral Computational Fluid Dynamics Using an Automatic Differentiation Adjoint," AIAA Journal, Vol 50, No. 12, 2012, pp. 2809-2819. doi:10.2514/1.J051658

[53] Theodorsen, T., "General Theory of Aerodynamic Instability and the Mechanism of Flutter," National Advisory Committee for Aeronautics, TR-496, 1935.

[54] Schütte, A., Hummel, D., and Hitzel, S. M., "Numerical and Experimental Analyses of the Vortical Flow Around the SACCON Configuration," AIAA Paper 2010-4690, 2010.

[55] Spalart, P., and Allmaras, S., "A One Equation Turbulence Model for Aerodynamic Flows," AIAA Paper 1992-0439, 1992.

[56] Rohlf, D., Schmidt, S., and Irving, J., "SACCON Stability and Control Analysis Applying System Identification Techniques," AIAA Paper 2010-4399, 2010

[57] Hicken, J. E., and Zingg, D. W., "Induced-Drag Minimization of Nonplanar Geometries Based on the Euler Equations," AIAA Journal, Vol 48, No. 11, 2010, pp. 2564-2575. doi:10.2514/1.J050379

[58] Lambe, A. B., and Martins, J. R. R. A., "Extensions to the Design Structure Matrix for the Description of Multidisciplinary Design, Analysis, and Optimization Processes," Structural and Multidisciplinary Optimization, Vol 46, No. 2, 2012, pp. 273-284. doi:10.1007/s00158-012-0763-y

W. K. Anderson Associate Editor 